\title{
Novel Small Organic Molecules for Highly Enantioselective Direct Aldol Reaction
}

\author{
Zhuo Tang, ${ }^{\text {a,b }}$ Fan Jiang, ${ }^{c}$ Luo-Ting Yu ${ }^{b}$, Xin Cui, ${ }^{a}$ Liu-Zhu Gong, ${ }^{\text {a }}$ Ai-Qiao, Mi, ${ }^{a}$ \\ Yao-Zhong Jiang ${ }^{\mathrm{a}}$, Yun-Dong $\mathrm{Wu}^{*^{\mathrm{c}}}$ \\ a. Key Laboratory for Asymmetric Synthesis of Sichuan Province, Chengdu Institute of \\ Organic Chemistry, Chinese Academy of Sciences, Chengdu, 610041, China. \\ b. Department of Applied Chemistry, College of Chemical Engineering, Sichuan \\ University, Chengdu, 610015, China \\ c. State Key Laboratory of Molecular Dynamics and Stable Structures, College of Chemistry and \\ Molecular Engineering, Peking University, China
}

\section{(Supporting information)}

General: NMR spectra were recorded on a Brucker-300 MHz spectrometer. Elemental analysis was carried out using Carlo Erba-1106 Analyzer. Optical rotations were measured on a Perkin-Elmer 241 Polarimeter at $=589 \mathrm{~nm}$. FT-ICRMS spectra were recorded on P-SIMS-Gly of Bruker Daltonics.Inc.. HPLC analysis was performed on Waters-Breeze (2487 Dual $\lambda$ Absorbance Detector and 1525 Binary HPLC Pump). Chiralpak AS, AD and OJ columns were purchased from Daicel Chemical Industries, LTD. Chiral GC analysis was performed on VARIAN CP-3380 with a CP CHIPASIL-DEX column. Acetone was dried over anhydrous $\mathrm{K}_{2} \mathrm{CO}_{3}$. Hexane and ethyl acetate for column chromatography were distilled before use.

Materials: All starting materials were purchased from Acros and used directly.

The typical procedure for the preparation of $1 \mathrm{a}-2 \mathrm{~b}$

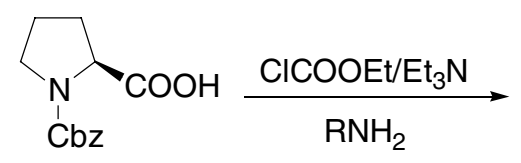

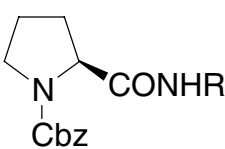

Z-A

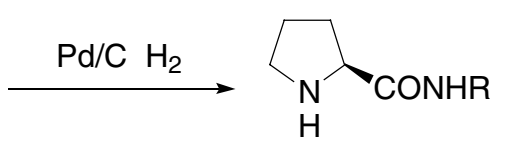

A

Typical procedure for the synthesis of $\mathbf{Z}$-A: $\boldsymbol{N}$-Carbobenzyloxy-L-proline $(2.0 \mathrm{~g}, 8.0 \mathrm{mmol})$ and TEA 
$(0.81 \mathrm{~g}, 8.0 \mathrm{mmol})$ were dissolved in THF $(30 \mathrm{~mL})$. The solution was cooled down to $0^{\circ} \mathrm{C}$. To the solution was added ethylchloroformate $(0.88 \mathrm{~g}, 8.0 \mathrm{mmol})$ dropwise for $15 \mathrm{~min}$. After the solution was stirred for $30 \mathrm{~min}$, amine $(8.0 \mathrm{mmol})$ was added for $15 \mathrm{~min}$. The resulting solution was stirred at $0{ }^{\circ} \mathrm{C}$ for $1 \mathrm{~h}$ and at room temperature for another $16 \mathrm{~h}$, and then refluxed for $3 \mathrm{~h}$. After cooled down to room temperature, the solution was diluted with ethyl acetate. After filtration and removal of solvent under reduced pressure, the residue was purified through column chromatography on silica gel (eluent: hexane: ethyl acetate $=2: 1$ ) to give Z-A .

Typical procedure for the synthesis of A: Compounds Z-A (1.0 g), 5\% Pd/C (0.1 g) and methanol (30 $\mathrm{ml})$ were mixed in a $100 \mathrm{~mL}$ two-neck flask. After stirred under hydrogen $(1 \mathrm{~atm})$ for $1 \mathrm{~h}$, the solution was filtered. Removal of solvent, the resulting residue was purified through column chromatography on silica gel (eluent: hexane: ethyl acetate $=2: 1$ ) to give $\mathbf{A}$.

(2S,1'R)-Pyrrolidine-2-carboxylic acid (1-benzyl-2-hydroxy-ethyl)- amide (1a) Yield: $79 \% \mathrm{mp}$ 136.9-138.7 ${ }^{0} \mathrm{C} ;[]^{20}{ }_{\mathrm{D}}=-16.6\left(\mathrm{c}=0.48, \mathrm{CH}_{3} \mathrm{CH}_{2} \mathrm{OH}\right) ;{ }^{1} \mathrm{HNMR}\left(300 \mathrm{MHz}, \mathrm{CDCl}_{3}\right) \quad$ (ppm) 1.65-1.72 (m, 2H), 1.94-2.15 (m, 3H), $1.87(\mathrm{~m}, 1 \mathrm{H}), 2.11(\mathrm{~m}, 1 \mathrm{H}), 2.52$ (br, 1H) 2.80-2.97 (m, 4H), 3.60-3.75 (m, 3H), $4.02(\mathrm{~m}, 1 \mathrm{H}), 7.21-7.34(\mathrm{~m}, 5 \mathrm{H}), 7.87$ (br, 1H); ${ }^{13} \mathrm{CNMR}\left(75 \mathrm{MHz}, \mathrm{CDCl}_{3}\right) \quad$ (ppm) 25.9, 30.7, 36.9, 47.0, 53.5, 60.4, 65.1, 126.5, 128.4, 129.1, 137.7, 175.8. HRMS (FT-ICRMS) exact mass calcd for $\left(\mathrm{C}_{14} \mathrm{H}_{20} \mathrm{~N}_{2} \mathrm{O}_{2}+\mathrm{H}\right)^{+}$requires $\mathrm{m} / \mathrm{z} 249.1603$, found $\mathrm{m} / \mathrm{z} 249.1585$.

(2S,1'S)-Pyrrolidine-2-carboxylic acid (1-benzyl-2-hydroxy-ethyl)- amide (2a): Yield: $82 \% \mathrm{mp}$ 104.4-105.9 ${ }^{0} \mathrm{C}$; [ ] ${ }_{\mathrm{D}}^{25}=-63.0\left(\mathrm{c}=0.5, \mathrm{CH}_{3} \mathrm{CH}_{2} \mathrm{OH}\right) ;{ }^{1} \mathrm{HNMR}\left(300 \mathrm{MHz}, \mathrm{CDCl}_{3}\right) \quad$ (ppm) $1.43(\mathrm{~m}, 2 \mathrm{H})$, $1.56(\mathrm{~m}, 3 \mathrm{H}), 1.71(\mathrm{~m}, 1 \mathrm{H}), 2.00(\mathrm{~m}, 1 \mathrm{H}), 2.78(\mathrm{~m}, 1 \mathrm{H}), 2.88-2.95(\mathrm{~m}, 2 \mathrm{H}), 3.60-3.76(\mathrm{~m}, 3 \mathrm{H}), 4.12(\mathrm{~m}$, 1H), 7.11-7.33 (m, 5H), 7.87 (br, 1H); ${ }^{13} \mathrm{CNMR}\left(75 \mathrm{MHz}, \mathrm{CDCl}_{3}\right) \quad$ (ppm) 26.7, 31.4, 37.9, 47.9, 53.9, 61.2, 66.5, 127.4, 129.3, 130.0, 138.5, 177.2; MS (ESI): $249.2\left(\mathrm{MH}^{+}\right)$. HRMS (FT-ICRMS) exact mass calcd for $\left(\mathrm{C}_{14} \mathrm{H}_{20} \mathrm{~N}_{2} \mathrm{O}_{2}+\mathrm{H}\right)^{+}$requires $\mathrm{m} / \mathrm{z} 249.1603$, found $\mathrm{m} / \mathrm{z} 249.1598$.

(2S,1'R)-Pyrrolidine-2-carboxylic acid (1-isopropyl-2-hydroxy-ethyl)-amide (1b): Yield: $85 \%$; mp 106.4-108.6 ${ }^{0}$; [ ] ${ }_{\mathrm{D}}^{20}=-25.1\left(\mathrm{c}=0.47, \mathrm{CH}_{3} \mathrm{CH}_{2} \mathrm{OH}\right) ;{ }^{1} \mathrm{HNMR}\left(300 \mathrm{MHz}, \mathrm{CDCl}_{3}\right) \quad$ (ppm) $0.95(\mathrm{~m}, 6 \mathrm{H})$, 1.70-1.76 (m, 2H), 1.93 (m, 2H), 2.12 (m, 1H), 2.56 (br, 1H), 2.90-3.01 (m, 2H), , 3.62 (m, 2H), 3.73 (m, 2H), 7.90 (br, 1H); ${ }^{13} \mathrm{CNMR}\left(75 \mathrm{MHz}, \mathrm{CDCl}_{3}\right) \quad$ (ppm) 18.4, 19.4, 26.0, 28.9, 30.8, 47.1, 57.6, 60.4, 64.5, 176.2. HRMS (FT-ICRMS) exact mass calcd for $\left(\mathrm{C}_{10} \mathrm{H}_{20} \mathrm{~N}_{2} \mathrm{O}_{2}+\mathrm{H}\right)^{+}$requires 201.1603, found $\mathrm{m} / \mathrm{z} 201.1590$. 
(2S,1'S)-Pyrrolidine-2-carboxylic acid (1-isopropyl-2-hydroxy-ethyl)-amide (2b): Yield: 94\%; mp 79.2-82.8 ${ }^{0} \mathrm{C}$; [ ] ${ }_{\mathrm{D}}^{25}=-83.4\left(\mathrm{c}=1.1, \mathrm{CH}_{3} \mathrm{CH}_{2} \mathrm{OH}\right) ;{ }^{1} \mathrm{HNMR}\left(300 \mathrm{MHz}, \mathrm{CDCl}_{3}\right) \quad$ (ppm) $0.93(\mathrm{~m}, 6 \mathrm{H})$, $1.72(\mathrm{~m}, 2 \mathrm{H}), 1.92(\mathrm{~m}, 2 \mathrm{H}), 2.20(\mathrm{~m}, 1 \mathrm{H}), 2.70(\mathrm{br}, 1 \mathrm{H}), 2.90-3.11(\mathrm{~m}, 2 \mathrm{H}), 3.60-3.80(\mathrm{~m}, 4 \mathrm{H}), 7.92(\mathrm{br}$, $1 \mathrm{H}) ;{ }^{13} \mathrm{CNMR}\left(75 \mathrm{MHz}, \mathrm{CDCl}_{3}\right) \quad$ (ppm)19.2, 20.5, 27.1, 29.8, 31.6, 48.1, 58.0, 61.4, 65.6, 177.4; HRMS (FT-ICRMS) exact mass calcd for $\left(\mathrm{C}_{10} \mathrm{H}_{20} \mathrm{~N}_{2} \mathrm{O}_{2}+\mathrm{H}\right)^{+}$requires 201.1603, found $\mathrm{m} / \mathrm{z}$ 201.1591.

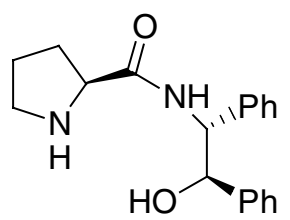

$3 a$

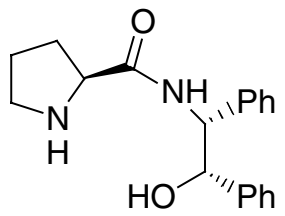

$3 \mathbf{b}$

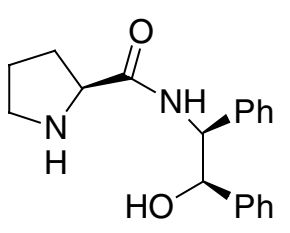

3c

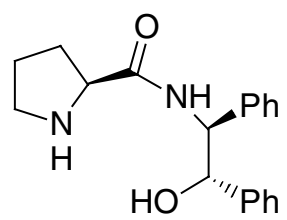

3d

Preparation and characterization of 3a: $N$-Carbobenzyloxy-L-proline $(2.0 \mathrm{~g}, 8.0 \mathrm{mmol})$ and TEA $(0.81 \mathrm{~g}, 8.0 \mathrm{mmol})$ were dissolved in THF $(30 \mathrm{ml})$. The solution was cooled down to $0^{\circ} \mathrm{C}$. To the solution was added ethylchloroformate $(0.88 \mathrm{~g}, 8.0 \mathrm{mmol})$ dropwise for $15 \mathrm{~min}$. After the solution was stirred for $30 \mathrm{~min},(1 R, 2 R)$-2-amino-1,2-diphenyl-ethanol (1.7 $\mathrm{g}, 8.0 \mathrm{mmol})$ was added for $15 \mathrm{~min}$. The resulting solution was stirred at $0{ }^{\circ} \mathrm{C}$ for $1 \mathrm{~h}$ and at room temperature for $16 \mathrm{~h}$, and refluxed for $3 \mathrm{~h}$. After the solution was cooled down to room temperature, the solution was washed with ethyl acetate, and filtered. After the solvent was evaporated to dryness, the residue was purified through column chromatography on a silica gel (eluent: hexane: ethyl acetate $=2: 1$ ) to give Z-3a.

Compounds Z-3a $(1.0 \mathrm{~g}), 5 \% \mathrm{Pd} / \mathrm{C}(0.1 \mathrm{~g})$ and methanol $(30 \mathrm{ml})$ were put in a $100 \mathrm{ml}$ two-neck flask. The solution was stirred under hydrogen at $50{ }^{\circ} \mathrm{C}$ for $2 \mathrm{~h}$, and filtered on Celite to remove any solids, then evaporated to dryness to give pure $\mathbf{3 a}$.

(2S,1'S,2'R)-Pyrrolidine-2-carboxylic acid (2-hydroxy-1,2-diphenyl-ethyl)-amide (3a):Yield: $82 \%$ mp 183.8-186.4 ${ }^{0} \mathrm{C}$ (Dec); [ $]^{23}{ }_{\mathrm{D}}=-29.2\left(\mathrm{c}=0.5, \mathrm{CH}_{3} \mathrm{CH}_{2} \mathrm{OH}\right) ;{ }^{1} \mathrm{HMNR}\left(300 \mathrm{MHz}, \mathrm{CD}_{3} \mathrm{OD}\right) \quad$ (ppm) 1.51-1.65 (m,3H), $2.95(\mathrm{~m}, 1 \mathrm{H}), 3.65(\mathrm{~m}, 1 \mathrm{H}), 4.99(\mathrm{~d}, J=4.2 \mathrm{~Hz}, 1 \mathrm{H}), 5.08(\mathrm{~d}, J=4.2 \mathrm{~Hz}, 1 \mathrm{H})$, 7.20-7.40 (m, 10H); ${ }^{13} \mathrm{CNMR}\left(75 \mathrm{MHz}, \mathrm{CDCl}_{3}\right.$ ) (ppm) 25.9, 30.5, 47.1, 59.0, 60.4, 77.1, 126.0, 126.9, 127.4, 127.5, 127.9, 128.5, 139.3, 140.7, 175.5. HRMS (FT-ICRMS) exact mass calcd for $\left(\mathrm{C}_{19} \mathrm{H}_{22} \mathrm{~N}_{2} \mathrm{O}_{2}\right.$ $+\mathrm{H})^{+}$requires $\mathrm{m} / \mathrm{z} 311.1760$, found $\mathrm{m} / \mathrm{z} 311.1750$. 
$\mathrm{N}$-Carbobenzyloxy-L-proline $(2.0 \mathrm{~g}, 8.0 \mathrm{mmol})$ and TEA $(0.81 \mathrm{~g}, 8.0 \mathrm{mmol})$ were dissolved in THF (30 $\mathrm{mL})$. The solution was cooled down to $0^{\circ} \mathrm{C}$. To the solution was added ethylchloroformate $(0.88 \mathrm{~g}, 8.0$ mmol) dropwise for 15 min. After the solution was stirred for 30 min, (1S,2R)-2-amino-1,2-diphenyl-ethanol (1.7 g, $8.0 \mathrm{mmol})$ was added for $15 \mathrm{~min}$. The resulting solution was stirred at $0{ }^{\circ} \mathrm{C}$ for $1 \mathrm{~h}$ and at room temperature for $16 \mathrm{~h}$, and then refluxed for $3 \mathrm{~h}$. After the solution was cooled down to room temperature, the solution was washed with ethyl acetate. After filtered, the solvent was evaporated and the residue was recrystallized from ethanol to give pure Z-3b

Compounds Z-3b $(1.0 \mathrm{~g}), 5 \% \mathrm{Pd} / \mathrm{C}(0.1 \mathrm{~g})$ and methanol $(30 \mathrm{~mL})$ were put in a $100 \mathrm{ml}$ two neck flask. The solution was stirred under hydrogen $(1 \mathrm{~atm})$ at $50{ }^{0} \mathrm{C}$ for $2 \mathrm{~h}$. After filtered, the solvent was evaporated to provide pure 3b. Yield: $70 \% \mathrm{mp} 201.1-203.6{ }^{0} \mathrm{C}(\mathrm{Dec})$; [ ] ${ }_{\mathrm{D}}^{23}=-28.4\left(\mathrm{c}=0.49 \mathrm{CH}_{3} \mathrm{OH}\right)$; ${ }^{1} \mathrm{HNMR}\left(300 \mathrm{MHz}, \mathrm{CDCl}_{3}\right) \quad$ (ppm) 1.61-1.68 (m,2H), 1.87 (m, 1H), 2.09 (m, 1H), $2.82(\mathrm{~m}, 1 \mathrm{H}), 2.96$ (m, 1H), $3.69(\mathrm{~m}, 1 \mathrm{H}), 4.98(\mathrm{~d}, J=4.8 \mathrm{~Hz}, 1 \mathrm{H}), .5 .32(\mathrm{~m}, 1 \mathrm{H}), 7.05-7.32(\mathrm{~m}, 10 \mathrm{H}), 8.35$ (br, 1H); ${ }^{13} \mathrm{CNMR}\left(75 \mathrm{MHz}, \mathrm{CDCl}_{3}\right) \quad$ (ppm) 26.0, 30.6, 47.1, 59.1, 60.3, 126.9, 127.4, 127.6, 127.7, 128.2, 137.8, 139.5, 175.6. HRMS (FT-ICRMS) exact mass calcd for $\left(\mathrm{C}_{19} \mathrm{H}_{22} \mathrm{~N}_{2} \mathrm{O}_{2}+\mathrm{H}\right)^{+}$requires $\mathrm{m} / \mathrm{z}$ 311.1760, found $\mathrm{m} / \mathrm{z} 311.1758\left(\mathrm{MH}^{+}\right)$

\section{The procedure for the synthesis of $3 \mathrm{c}$ is similar as $3 \mathrm{~b}$}

(2S,1'R,2'S)-Pyrrolidine-2-carboxylic acid (2-hydroxy-1,2-diphenyl-ethyl)-amide (3c): Yield: 74\% mp 141.7-144.8 ${ }^{0} \mathrm{C}$; [ ] ${ }^{22} \mathrm{D}=-23.8\left(\mathrm{c}=0.52, \mathrm{CH}_{3} \mathrm{CH}_{2} \mathrm{OH}\right) ;{ }^{1} \mathrm{HNMR}\left(300 \mathrm{MHz}, \mathrm{CD}_{3} \mathrm{OD}\right) \quad$ (ppm) 1.37 (m,1H), $1.56(\mathrm{~m}, 1 \mathrm{H}), 1.66(\mathrm{~m}, 1 \mathrm{H}), 1.93(\mathrm{~m}, 1 \mathrm{H}), 2.93(\mathrm{~m}, 1 \mathrm{H}), 3.64(\mathrm{~m}, 1 \mathrm{H}), 4.94(\mathrm{~d}, J=6.9 \mathrm{~Hz}, 1 \mathrm{H})$, $5.16(\mathrm{~d}, J=6.9 \mathrm{~Hz}, 1 \mathrm{H}), 7.26(\mathrm{~m}, 10 \mathrm{H}) ;{ }^{13} \mathrm{CNMR}\left(75 \mathrm{MHz}, \mathrm{CD}_{3} \mathrm{OD}\right) \quad$ (ppm) 25.9, 31.2, 47.3, 59.3, 60.9, 76.8, 127.6, 127.8, 128.1, 128.44, 128.47, 128.5, 140.0, 142.2, 174.2. HRMS (FT-ICRMS) exact mass calcd for $\left(\mathrm{C}_{19} \mathrm{H}_{22} \mathrm{~N}_{2} \mathrm{O}_{2}+\mathrm{H}\right)^{+}$requires $\mathrm{m} / \mathrm{z} 311.1760$, found $\mathrm{m} / \mathrm{z} 311.1751\left(\mathrm{MH}^{+}\right)$

\section{The procedure for the synthesis of $3 d$ is similar as $3 b$.}

(2S,1'S,2'S)-Pyrrolidine-2-carboxylic acid (2-hydroxy-1,2-diphenyl- ethyl)-amide (3d): Yield: $80 \%$ $\operatorname{mp} 66.9-70.7{ }^{0} \mathrm{C}$; [ ] ${ }_{\mathrm{D}}^{25}=-23.75\left(\mathrm{c}=2.0, \mathrm{CH}_{3} \mathrm{CH}_{2} \mathrm{OH}\right) ;{ }^{1} \mathrm{HNMR}\left(300 \mathrm{MHz}, \mathrm{CDCl}_{3}\right) \quad$ (ppm) 1.60-1.67 $(\mathrm{m}, 2 \mathrm{H}), 1.76(\mathrm{~m}, 1 \mathrm{H}), 2.04(\mathrm{~m}, 1 \mathrm{H}), 2.50(\mathrm{br}, 2 \mathrm{H}), 2.85-2.98(\mathrm{~m}, 2 \mathrm{H}), 3.65(\mathrm{~m}, 1 \mathrm{H}), 4.96(\mathrm{~d}, J=5.1 \mathrm{~Hz}$, $1 \mathrm{H}), 5.09(\mathrm{~m}, 1 \mathrm{H}), 7.18-7.31(\mathrm{~m}, 10 \mathrm{H}), 8.49(\mathrm{~d}, J=5.8 \mathrm{~Hz} \mathrm{1H}) ;{ }^{13} \mathrm{CNMR}\left(75 \mathrm{MHz}, \mathrm{CDCl}_{3}\right) \quad$ (ppm) 25.1, $30.5,47.26,59.7,60.4,77.7,126.3,126.9,127.52,127.54,128.0,128.5,139.4,141.0,175.8$; HRMS (FT-ICRMS) exact mass calcd for $\left(\mathrm{C}_{19} \mathrm{H}_{22} \mathrm{~N}_{2} \mathrm{O}_{2}+\mathrm{H}\right)^{+}$requires m/z 311.1760, found m/z 311.1751 . 
General procedure for aldol reaction: To a mixture of anhydrous acetone $(1 \mathrm{~mL})$ was added the corresponding aldehyde $(0.5 \mathrm{mmol})$ and L-proline derivative $(20 \mathrm{~mol} \%)$ and the resulting mixture was stirred at $-25{ }^{\circ} \mathrm{C}$ for $24-48 \mathrm{~h}$. The reaction mixture was treated with saturated ammonium chloride solution, the layers were separated, and the aqueous layer was extracted with ethyl acetate, dried over anhydrous $\mathrm{MgSO}_{4}$. After removal of solvent, the residue was purified through flash column chromatography on a silica gel (eluent: hexane: ethyl acetate $=1: 3$ ) to give the pure adducts.

4-Hydroxy-4-(4'-nitrophenyl)-butan-2-one (4a) ${ }^{2}$ : Yield: 66\%; [ ] ${ }_{\mathrm{D}}^{18}=+61.6\left(\mathrm{c}=0.51, \mathrm{CHCl}_{3}\right)$; ${ }^{1} \mathrm{HNMR}\left(300 \mathrm{MHz}, \mathrm{CDCl}_{3}\right) \quad(\mathrm{ppm}) 2.21(\mathrm{~s}, 3 \mathrm{H}), 2.83(\mathrm{~m}, 2 \mathrm{H}), 3.56(\mathrm{~d}, J=3.2 \mathrm{~Hz}, 1 \mathrm{H}), 5.25(\mathrm{~m}, 1 \mathrm{H})$, $7.52(\mathrm{~d}, J=7.0 \mathrm{~Hz}, 2 \mathrm{H}), 8.20(\mathrm{~d}, J=7.0 \mathrm{~Hz}, 2 \mathrm{H})$. Enantiomeric excess: 93\%, determined by HPLC (Daicel Chiralpak AS-H, i-PrOH/ Hexane=30/ 70), UV 254nm, flow rate $1.0 \mathrm{~mL} / \mathrm{min}$. $R$-isomer, $t_{R}$ 13.0min and $S$-isomer, $t_{R} 16.3 \mathrm{~min}$.

4-Hydroxy-4-(4'-bromophenyl)-butan-2-one (4b) ${ }^{2}$ : Yield: 77\%; [ ] ${ }_{\mathrm{D}}^{18}=+53.3\left(\mathrm{c}=0.42, \mathrm{CHCl}_{3}\right)$; ${ }^{1} \mathrm{HNMR}\left(300 \mathrm{MHz}, \mathrm{CDCl}_{3}\right) \quad(\mathrm{ppm}) 2.20(\mathrm{~s}, 3 \mathrm{H}), 2.82(\mathrm{~m}, 2 \mathrm{H}), 3.40(\mathrm{~d}, J=3.0 \mathrm{~Hz}, 1 \mathrm{H}), 5.12(\mathrm{~m}, 1 \mathrm{H})$, $7.24(\mathrm{~d}, J=8.4 \mathrm{~Hz}, 2 \mathrm{H}), 7.47(\mathrm{~d}, J=8.4 \mathrm{~Hz}, 2 \mathrm{H})$. Enantiomeric excess: 90\%, determined by HPLC (Daicel Chiralpak AS-H, i-PrOH/ Hexane=15/ 85), UV 262nm, flow rate $1.0 \mathrm{~mL} / \mathrm{min}$. $R$-isomer, $t_{R} 11.4$ min and $S$-isomer, $t_{R} 13.7$ min.

4-Hydroxy-4-(4'-chlorophenyl)-butan-2-one (4c): Yield: 75\%; [ ] ${ }_{\mathrm{D}}^{18}=+62.7 \quad\left(\mathrm{c}=0.48, \mathrm{CHCl}_{3}\right)$; ${ }^{1} \mathrm{HNMR}\left(300 \mathrm{MHz}, \mathrm{CDCl}_{3}\right) \quad$ (ppm) 2.18(s,1H), 2.82(m,1H), 3.46(br,1H) 5.11(m,1H), 7.29(m,4H); Enantiomeric excess: 93\%, determined by HPLC (Daicel Chiralpak AS-H, i-PrOH/ Hexane=10/ 90), UV $254 \mathrm{~nm}$, flow rate $1.0 \mathrm{~mL} / \mathrm{min}$. $R$-isomer, $t_{R} 14.1 \mathrm{~min}$ and $S$-isomer, $t_{R} 17.6 \mathrm{~min}$.

4-Hydroxy-4-(2'-chlorophenyl)-butan-2-one (4d) ${ }^{2}$ : Yield: 83\%; [ ] ${ }_{\mathrm{D}}^{18}=+97.0\left(\mathrm{c}=0.34, \mathrm{CHCl}_{3}\right)$; ${ }^{1} \mathrm{H}$ NMR (300 MHz, $\left.\mathrm{CDCl}_{3}\right) \quad(\mathrm{ppm}) 2.22(\mathrm{~s}, 3 \mathrm{H}), 2.64-3.03(\mathrm{~m}, 2 \mathrm{H}), 3.61$ (br, 1H), $5.56(\mathrm{~m}, 1 \mathrm{H})$, 7.19-7.34 (m, 3H), $7.64(\mathrm{t}, 1 \mathrm{H})$. Enantiomeric excess: 85\%, determined by HPLC (Daicel Chiralpak AS-H, i-PrOH/ Hexane=8/ 92), UV 262nm, flow rate $1.0 \mathrm{~mL} / \mathrm{min} . R$-isomer, $t_{R} 17.9 \mathrm{~min}$ and $S$-isomer, $t_{R} 12.5 \mathrm{~min}$.

4-Hydroxy-4-phenyl-butan-2-one (4e): Yield: 51\%; []$_{\mathrm{D}}^{20}=+60.0\left(\mathrm{c}=0.67, \mathrm{CHCl}_{3}\right) ;{ }^{1} \mathrm{HNMR}(300$ $\left.\mathrm{MHz} \mathrm{CDCl}_{3}\right) \quad(\mathrm{ppm}) 2.21(\mathrm{~s}, 3 \mathrm{H}), 2.87(\mathrm{~m}, 2 \mathrm{H}), 3.32(\mathrm{~d}, J=3.0 \mathrm{~Hz}, 1 \mathrm{H}), 5.17(\mathrm{~m}, 1 \mathrm{H}), 7.27-7.38(\mathrm{~m}$, 
5H. Enantiomeric excess: 83\%, determined by HPLC (Daicel Chiralpak AS-H, i-PrOH/ Hexane=15/ $85)$, UV $257 \mathrm{~nm}$, flow rate $1.0 \mathrm{~mL} / \mathrm{min} . R$-isomer, $t_{R} 11.2 \mathrm{~min}$ and $S$-isomer, $t_{R} 12.5 \mathrm{~min}$.

(4R)-4-(1-Naphthyl)-4-hydroxy-2-butanone (4f): Yield: 76\%; [ ] ${ }^{20}=+74.3 \quad\left(\mathrm{c}=0.48, \mathrm{CHCl}_{3}\right)$; ${ }^{1} \mathrm{HNMR}\left(300 \mathrm{MHz}, \mathrm{CDCl}_{3}\right) \quad(\mathrm{ppm}) 2.24(\mathrm{~s}, 3 \mathrm{H}), 3.00(\mathrm{~m}, 1 \mathrm{H}), 3.45(\mathrm{~d}, J=3.3 \mathrm{~Hz}, 1 \mathrm{H}), 5.97(\mathrm{t} 1 \mathrm{H})$ 7.47-7.54 (m, 3H), $7.71(\mathrm{~d}, J=10.2 \mathrm{~Hz}, 1 \mathrm{H}), 7.81(\mathrm{~d}, J=11.4 \mathrm{~Hz}, 1 \mathrm{H}), 7.88(\mathrm{~m}, 1 \mathrm{H}) 8.00(\mathrm{~m}, 1 \mathrm{H})$; Enantiomeric excess: 81\%, determined by HPLC (Daicel Chiralpak AS-H, i-PrOH/ Hexane=15/ 85), UV $243 \mathrm{~nm}$, flow rate $1.0 \mathrm{~mL} / \mathrm{min}$. $R$-isomer, $t_{R} 12.6 \mathrm{~min}$ and $S$-isomer, $t_{R} 13.8 \mathrm{~min}$.

4-(2-Naphthyl)-4-hydroxy-2-butanone (4g): Yield: 93\%; [ $]^{18}{ }_{\mathrm{D}}=+58.8\left(\mathrm{c}=0.5, \mathrm{CHCl}_{3}\right)$; $\mathrm{HNMR}$ $\left(300 \mathrm{MHz}, \mathrm{CDCl}_{3}\right) \quad$ (ppm) $2.21(\mathrm{~s}, 3 \mathrm{H}), 2.95(\mathrm{~m}, 2 \mathrm{H}), 3.44(\mathrm{bs}, 1 \mathrm{H}), 5.34(\mathrm{~m}, 1 \mathrm{H}), 7.45(\mathrm{~m}, \mathrm{H}), 7.82$ (m, 4H); Enantiomeric excess: 84\%, determined by HPLC (Daicel Chiralpak AS-H, i-PrOH/ Hexane $=15 / 85$ ), UV $257 \mathrm{~nm}$, flow rate $1.0 \mathrm{~mL} / \mathrm{min}$. $R$-isomer, $t_{R} 19.8 \mathrm{~min}$ and $S$-isomer, $t_{R} 22.1 \mathrm{~min}$

4-Hydroxy-4-(4-methylphenyl)-butan-2-one (4h): Yield: 48\%; [ $]^{20}{ }_{\mathrm{D}}=+57.1 \quad\left(\mathrm{c}=0.35, \mathrm{CHCl}_{3}\right)$; ${ }^{1} \mathrm{HNMR}\left(300 \mathrm{MHz}, \mathrm{CDCl}_{3}\right) \quad$ (ppm) $2.20(\mathrm{~s}, 3 \mathrm{H}), 2.35(\mathrm{~s}, 1 \mathrm{H})$ 2.77-2.88 (m, 2H), $3.32(\mathrm{~s}, 1 \mathrm{H}), 5.25(\mathrm{~d}$, $J=8.7 \mathrm{~Hz}, 2 \mathrm{H}), 7.17(\mathrm{~d}, J=7.8 \mathrm{~Hz}, 2 \mathrm{H}) 7.26$ (d, $J=7.8 \mathrm{~Hz}, 2 \mathrm{H})$; Enantiomeric excess: 84\%, determined by HPLC (Daicel Chiralpak, AS-H, i-PrOH $/$ Hexane=15/ 85), UV $257 \mathrm{~nm}$, flow rate $1.0 \mathrm{~mL} / \mathrm{min}$. $R$-isomer, $t_{R} 10.1 \mathrm{~min}$ and $S$-isomer, $t_{R} 12.4 \mathrm{~min}$.

4-Hydroxy-4-(3'-nitro-phenyl)-butan-2-one (4i): Yield: 63\%; [ ] ${ }^{20}=+62.1 \quad\left(\mathrm{c}=0.35, \mathrm{CHCl}_{3}\right)$; ${ }^{1} \mathrm{HNMR}\left(300 \mathrm{MHz}, \mathrm{CDCl}_{3}\right) \quad$ (ppm) 2.23 (s, 3H), 2.89 (t, 2H), $3.70(\mathrm{br}, 1 \mathrm{H}), 5.52(\mathrm{~m}, 1 \mathrm{H}), 7.53(\mathrm{t}, 1 \mathrm{H})$, $7.71(\mathrm{~d}, J=7.5 \mathrm{~Hz}, 1 \mathrm{H}), 8.13(\mathrm{~m}, 1 \mathrm{H}) 8.24(\mathrm{~s}, 1 \mathrm{H})$; Enantiomeric excess: 87\%, determined by HPLC (Daicel Chiralpak OJ-H, i-PrOH/ Hexane= 20/ 80), UV 254nm flow rate $1.0 \mathrm{~mL} / \mathrm{min} . R$-isomer, $t_{R} 17.3$ $\min$ and $S$-isomer, $t_{R} 19.9 \mathrm{~min}$.

(4R)-4-(Cyclohexyl)-4-hydroxy-2-butanone (4j): Yield: 85\%; [ $]^{18}{ }_{\mathrm{D}}=+45.9$ (c= 0.24, $\left.\mathrm{CHCl}_{3}\right)$; ${ }^{1} \mathrm{HNMR}\left(300 \mathrm{MHz}, \mathrm{CDCl}_{3}\right.$ ) (ppm) 0.97-1.25 (m, 6H), 1.62-1.77 (m, 5H), $2.18(\mathrm{~s}, 3 \mathrm{H}), 2.55(\mathrm{~m}, 2 \mathrm{H})$, 2.89 (bs, 1H), 3.80 (m 1H); Enantiomeric excess: 97\%, determined by HPLC (Daicel Chiralpak AD, i-PrOH/ Hexane=10/90), UV 280nm flow rate $1.0 \mathrm{~mL} / \mathrm{min}$. $R$-isomer, $t_{R} 6.1 \mathrm{~min}$ and $S$-isomer, $t_{R} 7.0$ $\min$. 
4-Hydroxy-5-methyl-hexan-2-one (4k): Yield: 43\%; [ ] ${ }^{18}=+77.6\left(\mathrm{c}=0.51, \mathrm{CHCl}_{3}\right) ;{ }^{1} \mathrm{HNMR}(300$ $\left.\mathrm{MHz}, \mathrm{CDCl}_{3}\right) \quad(\mathrm{ppm}) 0.90(\mathrm{~d}, J=6.6 \mathrm{~Hz}, 1 \mathrm{H}), 1.11(\mathrm{~m}, 1 \mathrm{H}), 1.43(\mathrm{~m}, 1 \mathrm{H}), 1.77(\mathrm{~m}, 1 \mathrm{H}), 2.17(\mathrm{~s}, 1 \mathrm{H})$, $2.56(\mathrm{~m}, 2 \mathrm{H}), 2.97$ (br, 1H), 4.11(m, 1H); Enantiomeric excess: 98\%, determined by HPLC (Daicel Chiralpak AD, i-PrOH/ Hexane=3/ 97), UV 280nm flow rate $1.0 \mathrm{~mL} / \mathrm{min} . R$-isomer, $t_{R} 9.1 \mathrm{~min}$ and $S$-isomer, $t_{R} 10.0 \mathrm{~min}$.

4-Hydroxy-5,5-dimethyl-hexan-2-one (4I): Yield: 51\%; [ $]^{18}{ }_{\mathrm{D}}=+75.5\left(\mathrm{c}=0.38, \mathrm{CHCl}_{3}\right)$; ${ }^{1} \mathrm{HNMR}$ (300 MHz, $\left.\mathrm{CDCl}_{3}\right) \quad$ (ppm) 0.91(s,1H), 2.52-2.66 (m,2H), 3.77(m,1H); Enantiomeric excess: >99\%, determined by HPLC (Daicel Chiralpak AD, i-PrOH/ Hexane=3/ 97), UV 280nm flow rate 1.0 $\mathrm{mL} / \mathrm{min} . R$-isomer, $t_{R} 7.4 \mathrm{~min}$ and $S$-isomer, $t_{R} 8.8 \mathrm{~min}$.

4-Hydroxy-heptan-2-one (4m): Yield: $17 \%$; ${ }^{1} \mathrm{HNMR}\left(300 \mathrm{MHz}, \mathrm{CDCl}_{3}\right) \quad$ (ppm) 0.93 (m, $\left.1 \mathrm{H}\right)$, 1.34-1.51(m, 2H) $2.18(\mathrm{~s}, 3 \mathrm{H}) 2.60(\mathrm{~m}, 1 \mathrm{H}) 2.97(\mathrm{br}, 1 \mathrm{H}) 4.08(\mathrm{~m}, 1 \mathrm{H})$; Enantiomeric excess: 87\%, determined by chiral GC analysis (CP CHIRASIL-DEX), Inject Temp $240{ }^{\circ} \mathrm{C}$, Column Temp $110{ }^{\circ} \mathrm{C}$, FID Oven Temp $260{ }^{\circ} \mathrm{C}$, Inlet Pressure 10 Psi. $R$-isomer, $t_{R} 5.7 \mathrm{~min}$ and $S$-isomer, $t_{R} 5.9 \mathrm{~min}$.

4-Hydroxy-octan-2-one (4n): Yield: $12 \%$; ${ }^{1} \mathrm{HNMR}\left(300 \mathrm{MHz}, \mathrm{CDCl}_{3}\right) \quad$ (ppm) 0.82 (t, J=7.2 Hz, $1 \mathrm{H}), 1.22-1.40(\mathrm{~m}, 4 \mathrm{H}) 2.10(\mathrm{~s}, 3 \mathrm{H}) 2.46-2.58(\mathrm{~m}, 2 \mathrm{H}) 2.88(\mathrm{br}, 1 \mathrm{H}) 3.95(\mathrm{~m}, 1 \mathrm{H})$; Enantiomeric excess: $87 \%$, determined by chiral GC analysis (CP CHIRASIL-DEX ), Inject Temp $240{ }^{\circ} \mathrm{C}$,Column Temp $120{ }^{\circ} \mathrm{C}$, FID Oven Temp $260{ }^{\circ} \mathrm{C}$, Inlet Pressure 10 Psi. $R$-isomer, $t_{R} 6.0 \mathrm{~min}$ and $S$-isomer, $t_{R} 6.2 \mathrm{~min}$. 


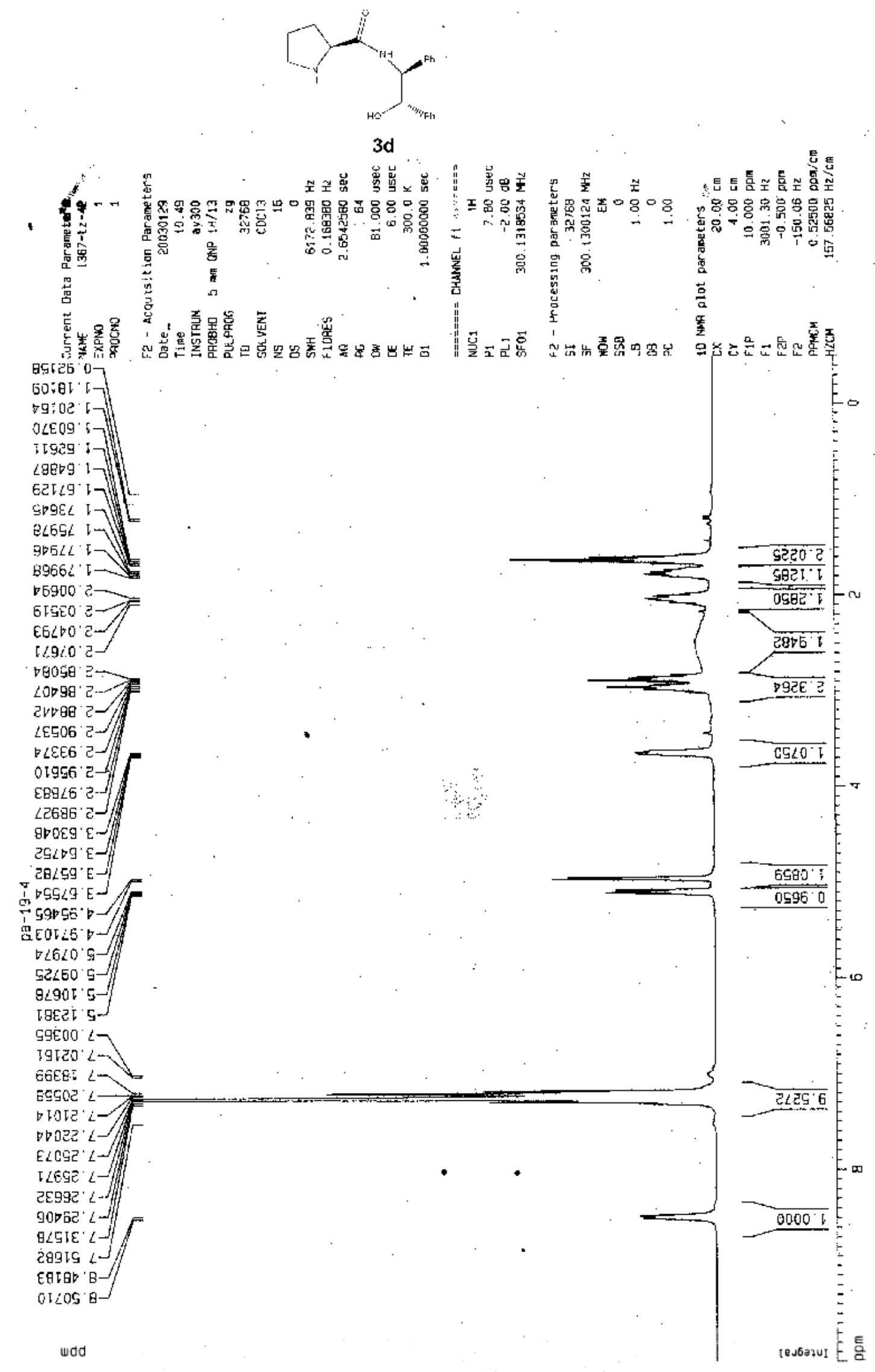


<smiles>CC(=O)CC(O)c1ccc([N+](=O)[O-])cc1</smiles>

(4a)-Racemic

ulas

Project Name: Waters

Reported by User. System

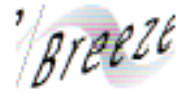

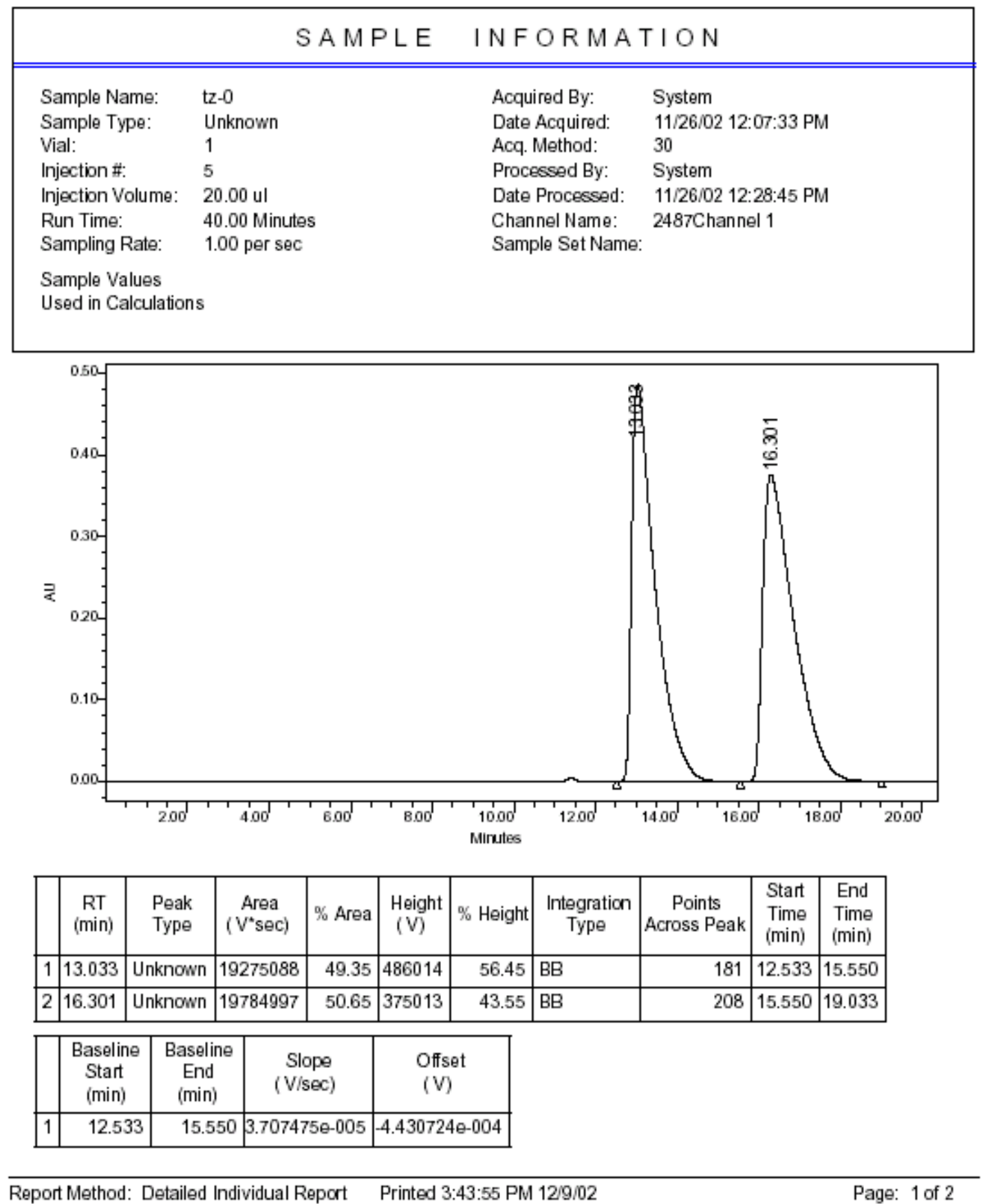


<smiles>CC(=O)CC(O)c1ccc([N+](=O)[O-])cc1</smiles>

(4a), $93 \%$ ee

ulas

Project Name: Waters

Reported by User. System

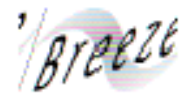

\begin{tabular}{|llll|}
\hline & & S A M P L E & I N F O R M A T I O N \\
\hline \hline Sample Name: & tz-89 & Acquired By: & System \\
Sample Type: & Unknown & Date Acquired: & $11 / 26 / 02$ \\
Vial: & 1 & Acq. Method: & 30 \\
Injection \#: & 2 & Processed By: & System \\
Injection Volume: & 20.00 ul & Date Processed: $11 / 26 / 0211: 56: 54$ AM \\
Run Time: & 40.00 Minutes & Channel Name: 2487 Channel 1 \\
Sampling Rate: & 1.00 per sec & Sample Set Name: \\
Sample Values & & \\
Used in Calculations & & \\
& & \\
\end{tabular}

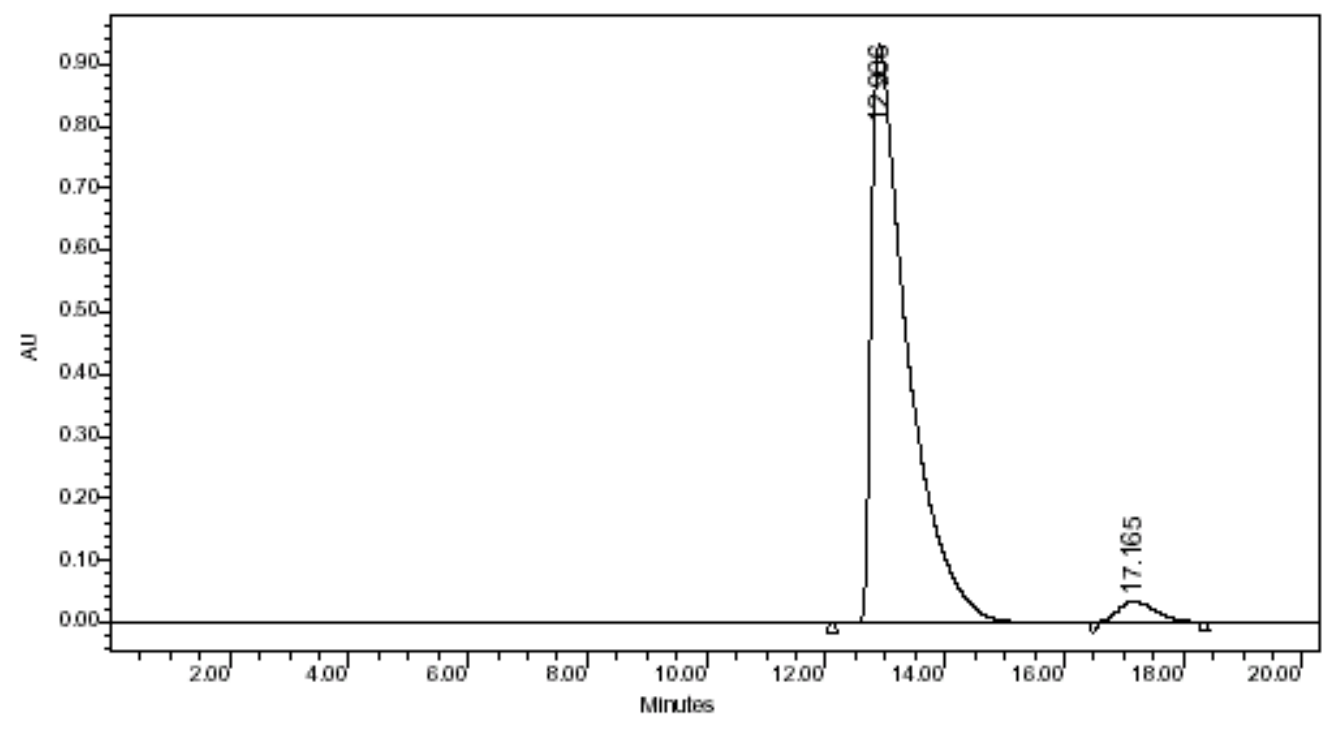

\begin{tabular}{|l|c|c|c|r|r|r|r|r|r|c|}
\hline & $\begin{array}{c}\mathrm{RT} \\
\text { (min) }\end{array}$ & $\begin{array}{c}\text { Peak } \\
\text { Type }\end{array}$ & $\begin{array}{c}\text { Area } \\
\text { (V'sec) }\end{array}$ & $\%$ Area & $\begin{array}{l}\text { Height } \\
\text { (V) }\end{array}$ & \% Height & $\begin{array}{l}\text { Integration } \\
\text { Type }\end{array}$ & $\begin{array}{c}\text { Points } \\
\text { Across Peak }\end{array}$ & $\begin{array}{c}\text { Start } \\
\text { Time } \\
\text { (min) }\end{array}$ & $\begin{array}{c}\text { End } \\
\text { Time } \\
\text { (min) }\end{array}$ \\
\hline 1 & 12.906 & Unknown & 39247715 & 96.33 & 931362 & 96.44 & bv & 263 & 12.117 & 16.500 \\
\hline 2 & 17.165 & Unknown & 1493391 & 3.67 & 34336 & 3.56 & vb & 112 & 16.500 & 18.367 \\
\hline
\end{tabular}

\begin{tabular}{|c|c|c|c|c|}
\hline & $\begin{array}{c}\text { Baseline } \\
\text { Start } \\
\text { (min) }\end{array}$ & $\begin{array}{c}\text { Baseline } \\
\text { End } \\
\text { (min) }\end{array}$ & $\begin{array}{c}\text { Slope } \\
\text { (V/sec) }\end{array}$ & $\begin{array}{c}\text { Offset } \\
\text { (V) }\end{array}$ \\
\hline 1 & 12.117 & 18.367 & $1.074371 \mathrm{e}-004$ & $-1.302841 \mathrm{e}-003$ \\
\hline
\end{tabular}

Report Method: Detailed Individual Report $\quad$ Printed 3:45:09 PM 12/9/02

Page: 1 of 2 
<smiles>CC(=O)CC(O)c1ccc(Br)cc1</smiles>

(4b)-racemic

ulas

Project Name: Waters

Reported by User. System

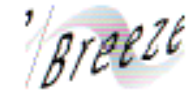

\begin{tabular}{|llll|}
\hline & \multicolumn{2}{c|}{ S A M P L E } & I N F O R M A T I O N \\
\hline \hline Sample Name: & tz-Br-0. & Acquired By: & System \\
Sample Type: & Unknown & Date Acquired: $12 / 9102$ 1:51:54 PM \\
Vial: & 1 & Acq. Method: 15 \\
Injection \#: & 2 & Processed By: & System \\
Injection Volume: & 20.00 ul & Date Processed: $12 / 9022: 09: 35$ PM \\
Run Time: & 50.00 Minutes & Channel Name: 2487Channel 1 \\
Sampling Rate: & 1.00 per sec & Sample Set Name: \\
Sample Values & & \\
Used in Calculations & & \\
& & \\
\end{tabular}

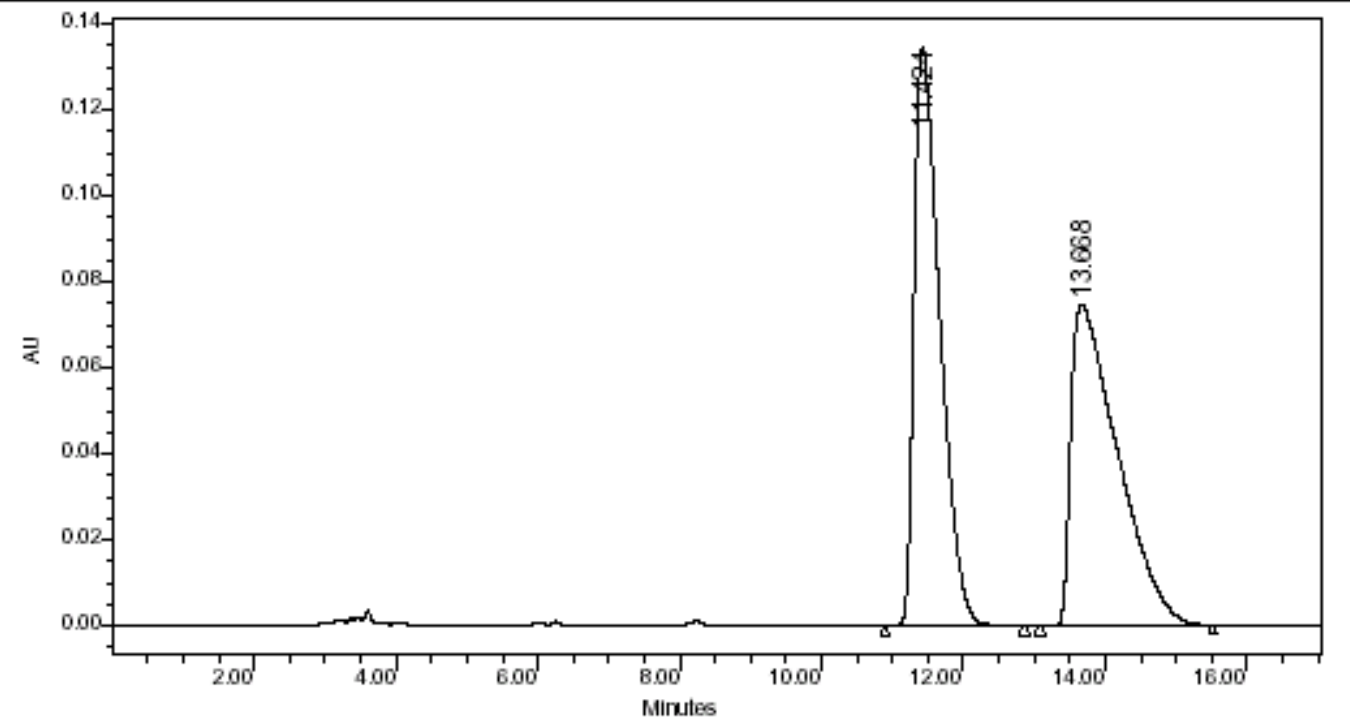

\begin{tabular}{|l|c|c|c|c|c|c|c|r|r|r|}
\hline & $\begin{array}{c}\text { RT } \\
\text { (min) }\end{array}$ & $\begin{array}{c}\text { Peak } \\
\text { Type }\end{array}$ & $\begin{array}{c}\text { Area } \\
\text { (V'sec) }\end{array}$ & $\%$ Area & $\begin{array}{c}\text { Height } \\
\text { (V) }\end{array}$ & $\%$ Height & $\begin{array}{l}\text { Integration } \\
\text { Type }\end{array}$ & $\begin{array}{c}\text { Points } \\
\text { Across Peak }\end{array}$ & $\begin{array}{c}\text { Start } \\
\text { Time } \\
\text { (min) }\end{array}$ & $\begin{array}{c}\text { End } \\
\text { Time } \\
\text { (min) }\end{array}$ \\
\hline 1 & 11.424 & Unknown & 3396586 & 49.87 & 134505 & 64.26 & BB & 118 & 10.900 & 12.867 \\
\hline 2 & 13.668 & Unknown & 3414883 & 50.13 & 74793 & 35.74 & BB & 146 & 13.083 & 15.533 \\
\hline
\end{tabular}

\begin{tabular}{|c|c|c|c|c|}
\hline & $\begin{array}{c}\text { Baseline } \\
\text { Start } \\
\text { (min) }\end{array}$ & $\begin{array}{c}\text { Baseline } \\
\text { End } \\
\text { (min) }\end{array}$ & $\begin{array}{c}\text { Slope } \\
\text { (V/sec) }\end{array}$ & $\begin{array}{c}\text { Offset } \\
\text { (V) }\end{array}$ \\
\hline 1 & 10.900 & 12.867 & $-2.715547 \theta-005$ & $3.601292 \Theta-004$ \\
\hline
\end{tabular}

Report Method: Detailed Individual Report $\quad$ Printed 2:58:19 PM 12/9/02

Page: 1 of 2 
<smiles>CC(=O)CC(O)c1ccc(Br)cc1</smiles>

(4b), $90 \%$ ee

ulas

Project Name: Waters

Reported by User. System

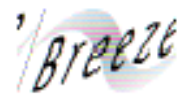

\begin{tabular}{|llll|}
\hline & \multicolumn{2}{c|}{ S A M PLE } & I N F O R M A T I O N \\
\hline \hline Sample Name: & tz-Br-TT-12 & Acquired By: & System \\
Sample Type: & Unknown & Date Acquired: & $12 / 9022: 10: 57$ PM \\
Vial: & 1 & Acq. Method: & 15 \\
Injection \#: & 3 & Processed By: & System \\
Injection Volume: & $20.00 \mathrm{ul}$ & Date Processed: & $12 / 9 / 024: 11: 13 \mathrm{PM}$ \\
Run Time: & 50.00 Minutes & Channel Name: & 2487Channel 1 \\
Sampling Rate: & 1.00 per sec & Sample Set Name: \\
Sample Values & & & \\
Used in Calculations & & \\
& & & \\
\end{tabular}

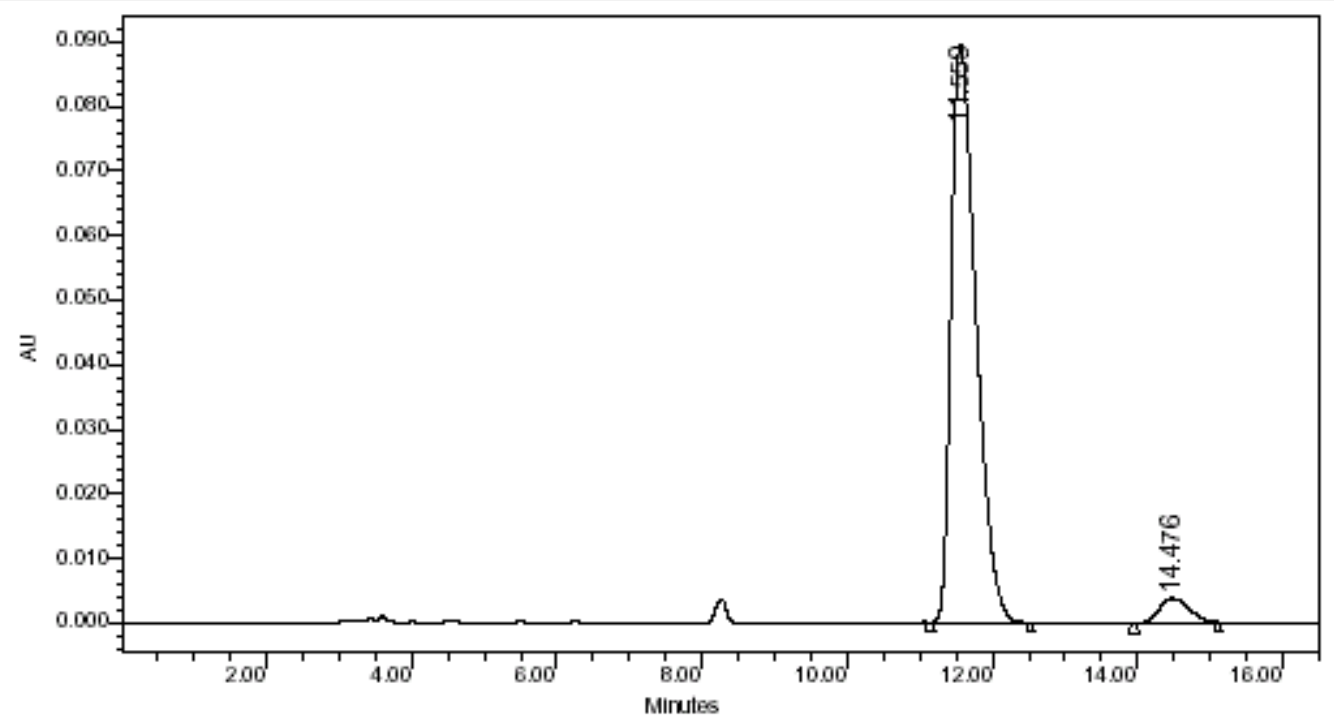

\begin{tabular}{|l|c|c|r|r|r|r|l|r|r|c|}
\hline & $\begin{array}{c}\text { RT } \\
\text { (min) }\end{array}$ & $\begin{array}{c}\text { Peak } \\
\text { Type }\end{array}$ & $\begin{array}{c}\text { Area } \\
\left(V^{*} \text { sec }\right)\end{array}$ & $\%$ Area & $\begin{array}{c}\text { Height } \\
\text { (V) }\end{array}$ & $\%$ Height & $\begin{array}{l}\text { Integration } \\
\text { Type }\end{array}$ & $\begin{array}{c}\text { Points } \\
\text { Across Peak }\end{array}$ & $\begin{array}{c}\text { Start } \\
\text { Time } \\
\text { (min) }\end{array}$ & $\begin{array}{c}\text { End } \\
\text { Time } \\
\text { (min) }\end{array}$ \\
\hline 1 & 11.559 & Unknown & 2116173 & 95.12 & 89424 & 95.92 & BB & 82 & 11.150 & 12.533 \\
\hline 2 & 14.476 & Unknown & 108592 & 4.88 & 3799 & 4.08 & Bb & 69 & 13.950 & 15.117 \\
\hline
\end{tabular}

\begin{tabular}{|c|c|c|c|c|}
\hline & $\begin{array}{c}\text { Baseline } \\
\text { Start } \\
\text { (min) }\end{array}$ & $\begin{array}{c}\text { Baseline } \\
\text { End } \\
\text { (min) }\end{array}$ & $\begin{array}{c}\text { Slope } \\
\text { (V/sec) }\end{array}$ & $\begin{array}{c}\text { Offset } \\
\text { (V) }\end{array}$ \\
\hline 1 & 11.150 & 12.533 & $-4.570742 \mathrm{e}-005$ & $6.769897 \Theta-004$ \\
\hline
\end{tabular}


<smiles>CC(=O)CC(O)c1ccc(Cl)cc1</smiles>

(4c )-racemic

ulas

Project Name: Defaults

'breezt

Reported by User: System

\begin{tabular}{|llll|}
\hline & \multicolumn{2}{c|}{ S A M P L E } & I N F O R M A T I O N \\
\hline \hline & & & \\
Sample Name: & TZ-140 & Acquired By: & System \\
Sample Type: & Unknown & Date Acquired: & $2 / 6 / 0310: 56: 54$ AM \\
Vial: & 1 & Acq. Method: & $100 \mathrm{~A}$ \\
Injection \#: & 1 & Date Processed: $2 / 6 / 0311: 24: 35 \mathrm{AM}$ \\
Injection Volume: & $20.00 \mathrm{ul}$ & Channel Name: 2487 Channel 1 \\
Run Time: & 60.00 Minutes & Sample Set Name: \\
\hline
\end{tabular}

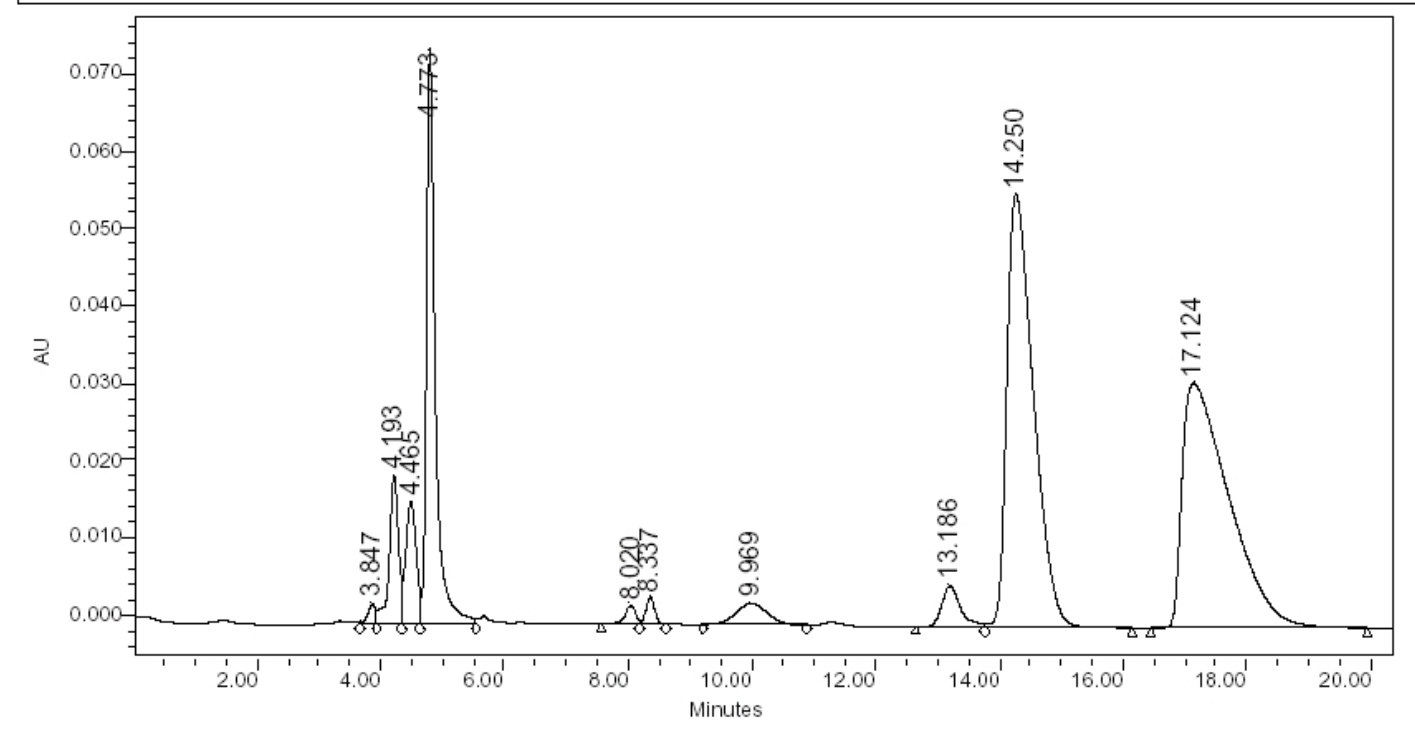

\begin{tabular}{|r|r|r|r|r|r|}
\hline & $\begin{array}{c}\text { RT } \\
(\mathrm{min})\end{array}$ & $\begin{array}{c}\text { Area } \\
\left(\mathrm{V}^{*} \mathrm{sec}\right)\end{array}$ & \% Area & $\begin{array}{c}\text { Height } \\
(\mathrm{V})\end{array}$ & $\begin{array}{c}\% \\
\text { Height }\end{array}$ \\
\hline 1 & 3.847 & 26093 & 0.55 & 2687 & 1.25 \\
\hline 2 & 4.193 & 202944 & 4.26 & 19303 & 8.99 \\
\hline 3 & 4.465 & 186396 & 3.91 & 15981 & 7.45 \\
\hline 4 & 4.773 & 739187 & 15.51 & 74804 & 34.86 \\
\hline 5 & 8.020 & 29277 & 0.61 & 2499 & 1.16 \\
\hline 6 & 8.337 & 34094 & 0.72 & 3534 & 1.65 \\
\hline 7 & 9.969 & 102990 & 2.16 & 2825 & 1.32 \\
\hline 8 & 13.186 & 113015 & 2.37 & 5278 & 2.46 \\
\hline 9 & 14.250 & 1667600 & 34.99 & 56050 & 26.12 \\
\hline 10 & 17.124 & 1664871 & 34.93 & 31642 & 14.74 \\
\hline
\end{tabular}


<smiles>CC(=O)CC(O)c1ccc(Cl)cc1</smiles>

(4c), $93 \%$ ee

ulas

Project Name: Defaults

Reported by User: System

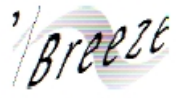

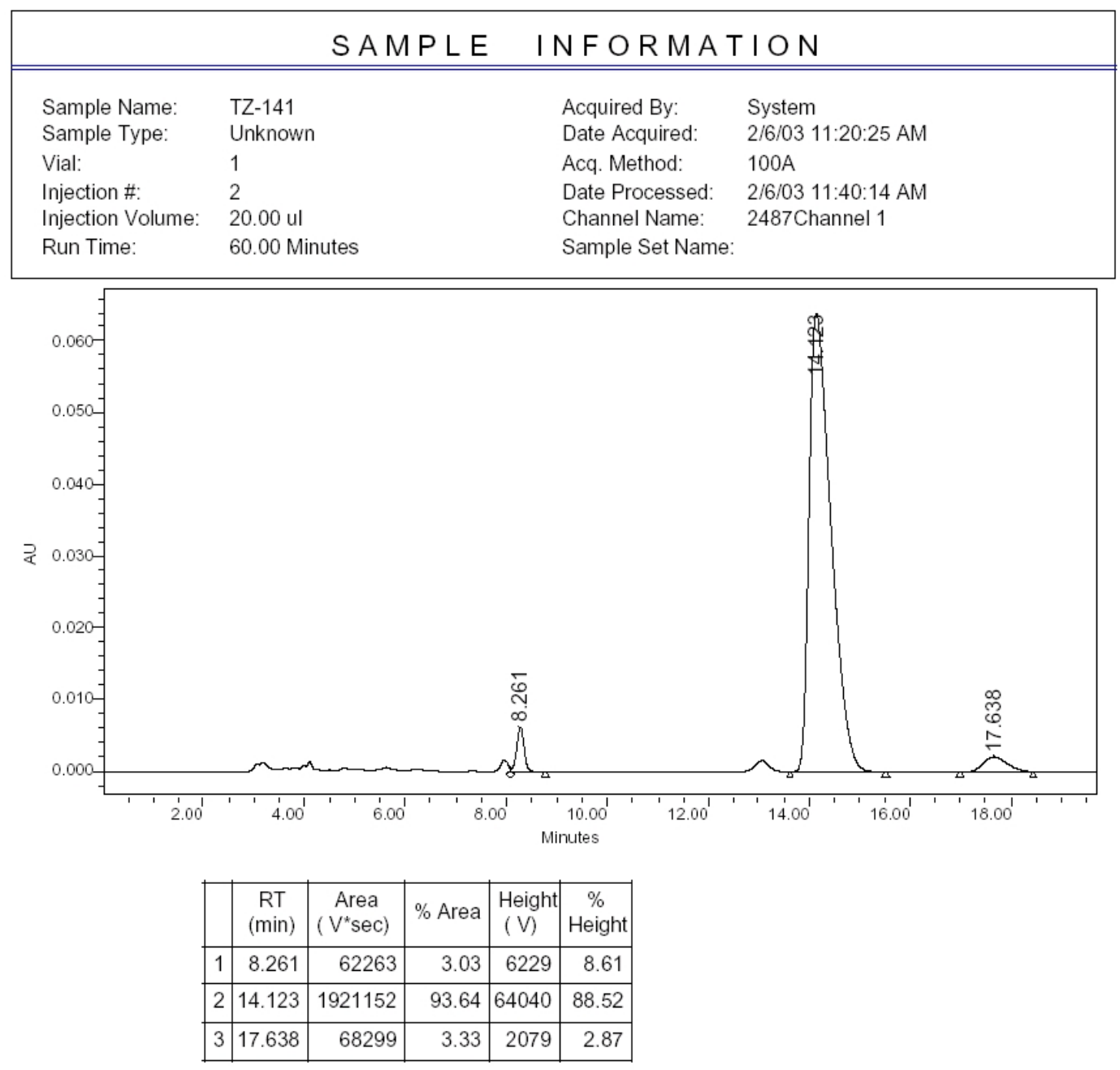


<smiles>CC(=O)CC(O)C1CCCCC1</smiles>

( 4j )- racemic

ulas

Project Name: Defaults Reported by User: System
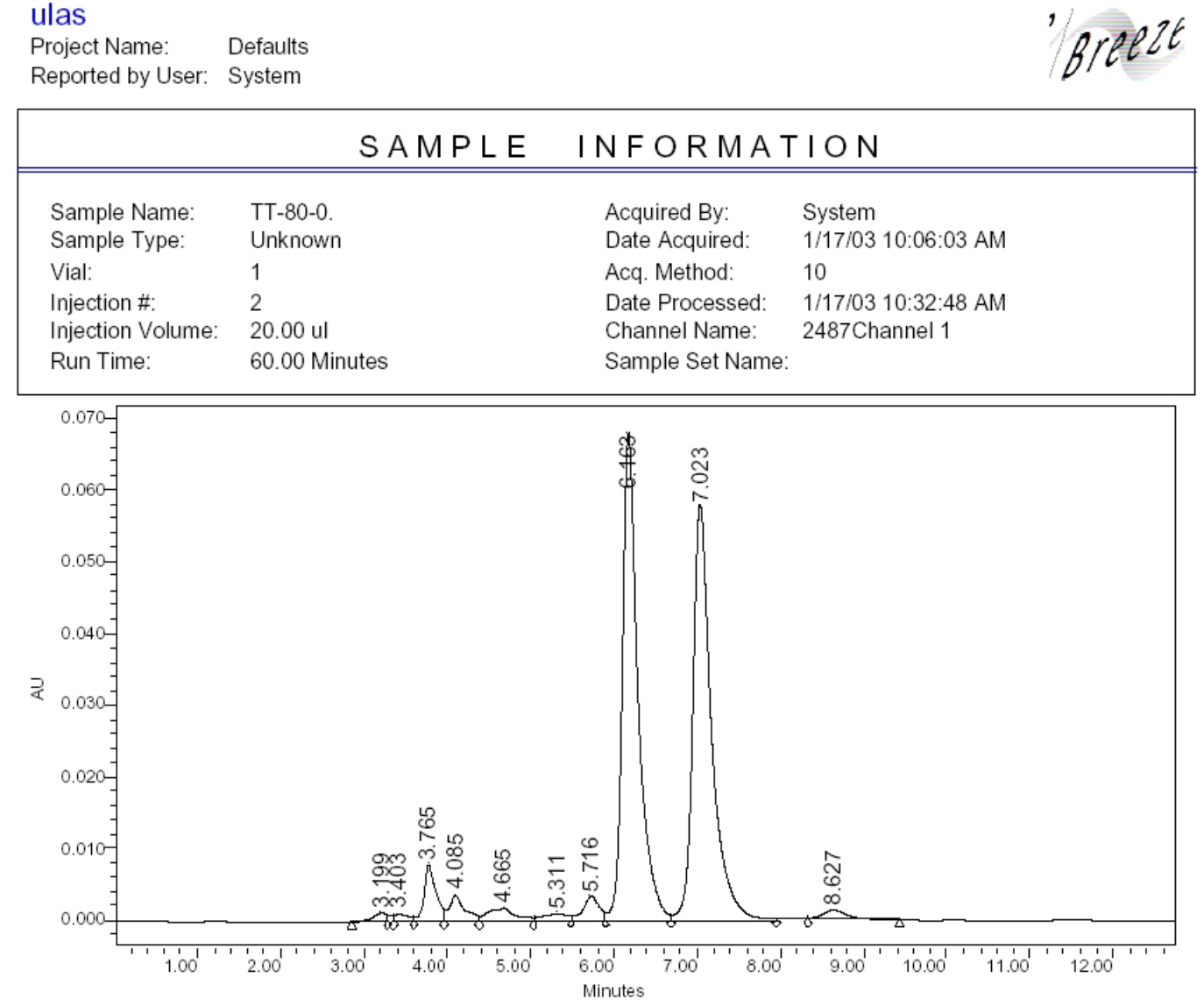

\begin{tabular}{|r|r|r|r|r|r|}
\hline & $\begin{array}{c}\text { RT } \\
(\mathrm{min})\end{array}$ & $\begin{array}{c}\text { Area } \\
\left(\mathrm{V}^{*} \mathrm{sec}\right)\end{array}$ & $\%$ Area & $\begin{array}{l}\text { Height } \\
(\mathrm{V})\end{array}$ & $\begin{array}{c}\% \\
\text { Height }\end{array}$ \\
\hline 1 & 3.199 & 14776 & 0.70 & 1314 & 0.89 \\
\hline 2 & 3.403 & 12855 & 0.61 & 1082 & 0.73 \\
\hline 3 & 3.765 & 78599 & 3.75 & 7891 & 5.33 \\
\hline 4 & 4.085 & 47610 & 2.27 & 3642 & 2.46 \\
\hline 5 & 4.665 & 42723 & 2.04 & 1912 & 1.29 \\
\hline 6 & 5.311 & 22410 & 1.07 & 1086 & 0.73 \\
\hline 7 & 5.716 & 47772 & 2.28 & 3476 & 2.35 \\
\hline 8 & 6.163 & 892686 & 42.55 & 68188 & 46.04 \\
\hline 9 & 7.023 & 905273 & 43.15 & 58077 & 39.22 \\
\hline 10 & 8.627 & 33172 & 1.58 & 1427 & 0.96 \\
\hline
\end{tabular}


<smiles>CC(=O)CC(O)C1CCCCC1</smiles>

( $\mathbf{j}$ ) $97 \%$ ee

ulas

Project Name: Defaults

Reported by User: System

\begin{tabular}{|llll|}
\hline & \multicolumn{2}{c|}{ S A M P L E } & I N F O R M A T I O N \\
\hline \hline & & & \\
Sample Name: & TT-82 & Acquired By: & System \\
Sample Type: & Unknown & Date Acquired: $1 / 17 / 0310: 51: 30 \mathrm{AM}$ \\
Vial: & 1 & Acq. Method: & 10 \\
Injection \#: & 5 & Date Processed: $1 / 17 / 0311: 01: 24 \mathrm{AM}$ \\
Injection Volume: & $20.00 \mathrm{ul}$ & Channel Name: & 2487Channel 1 \\
Run Time: & 60.00 Minutes & Sample Set Name: \\
\hline
\end{tabular}

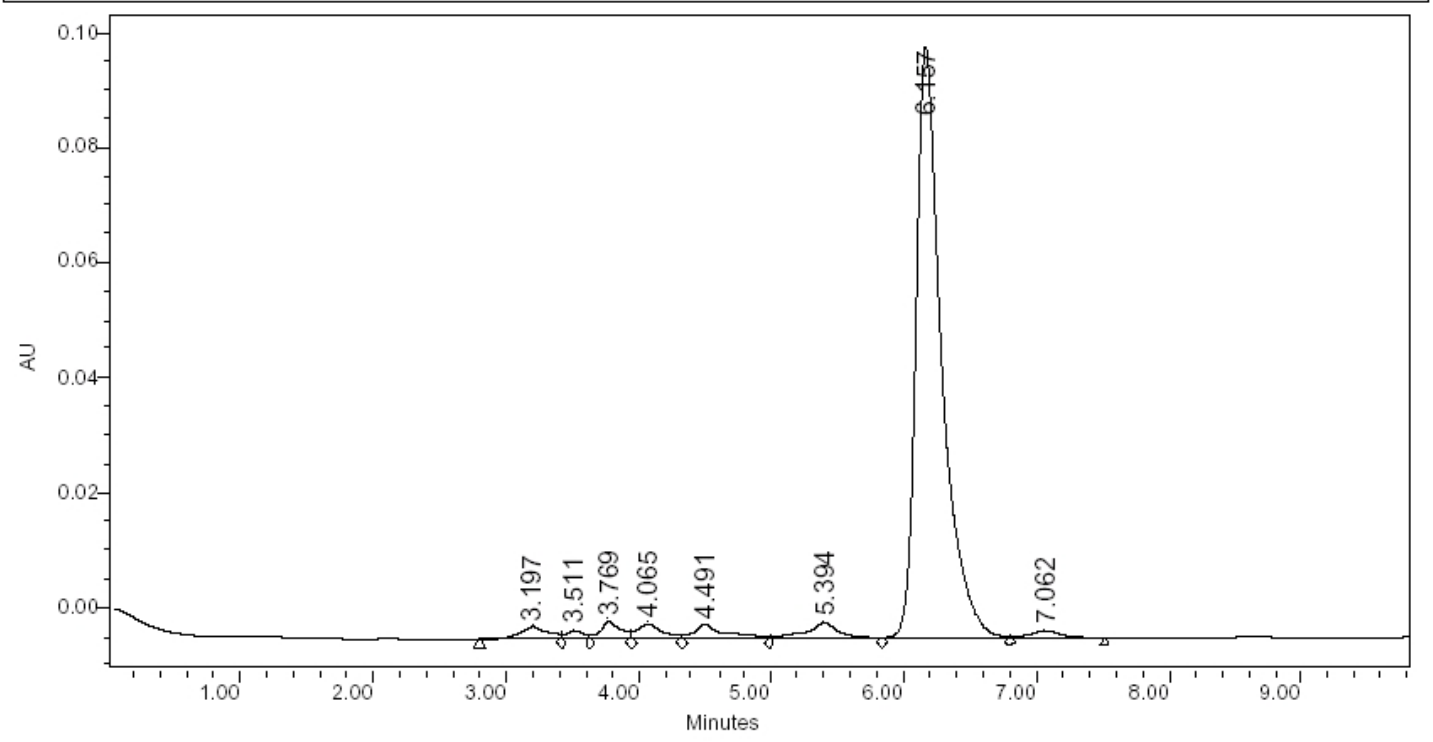

\begin{tabular}{|r|r|r|r|r|r|}
\hline & $\begin{array}{c}\text { RT } \\
(\mathrm{min})\end{array}$ & $\begin{array}{c}\text { Area } \\
\left(\mathrm{V}^{*} \mathrm{sec}\right)\end{array}$ & $\%$ Area & $\begin{array}{c}\text { Height } \\
(\mathrm{V})\end{array}$ & $\begin{array}{c}\% \\
\text { Height }\end{array}$ \\
\hline 1 & 3.197 & 32664 & 2.03 & 2182 & 1.83 \\
\hline 2 & 3.511 & 13543 & 0.84 & 1463 & 1.23 \\
\hline 3 & 3.769 & 33714 & 2.10 & 3094 & 2.60 \\
\hline 4 & 4.065 & 34268 & 2.13 & 2613 & 2.20 \\
\hline 5 & 4.491 & 42080 & 2.62 & 2496 & 2.10 \\
\hline 6 & 5.394 & 48703 & 3.03 & 2664 & 2.24 \\
\hline 7 & 6.157 & 1379364 & 85.89 & 103172 & 86.74 \\
\hline 8 & 7.062 & 21547 & 1.34 & 1265 & 1.06 \\
\hline
\end{tabular}




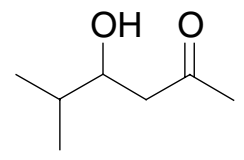

( 4k )-racemic

ulas

Project Name: Defaults

Reported by User: System

'breelt

\begin{tabular}{|llll|}
\hline \multicolumn{3}{c|}{ S A M P LE } & I N F O R M A T I O N \\
\hline \hline & & & \\
Sample Name: & TZ-90Unk. & Acquired By: & System \\
Sample Type: & Unknown & Date Acquired: & $1 / 20 / 0312: 56: 20 \mathrm{PM}$ \\
Vial: & 1 & Acq. Method: & 3 uv280 \\
Injection \#: & 1 & Date Processed: $1 / 20 / 031: 28: 13 \mathrm{PM}$ \\
Injection Volume: & $20.00 \mathrm{ul}$ & Channel Name: & 2487 Channel 1 \\
Run Time: & 60.00 Minutes & Sample Set Name: \\
\hline
\end{tabular}

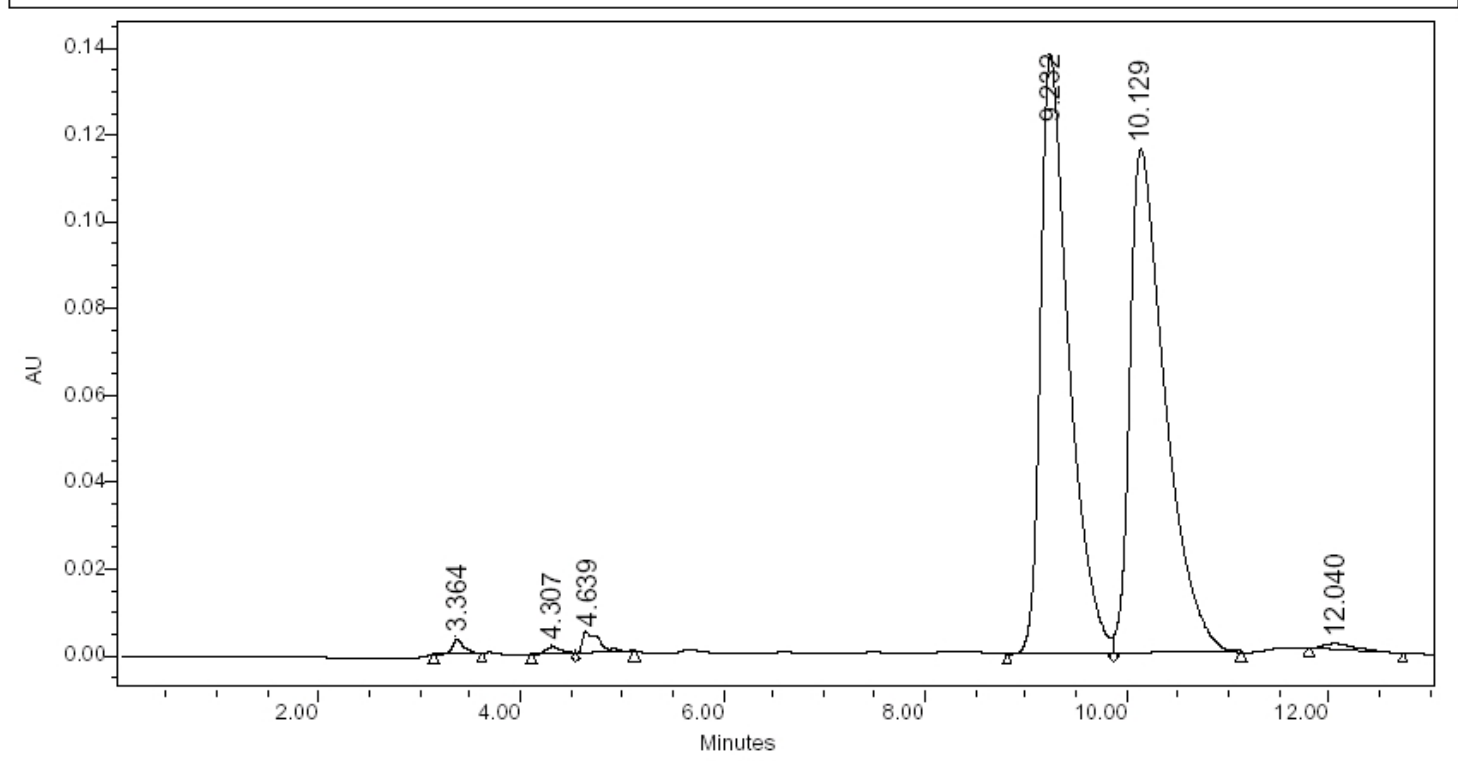

\begin{tabular}{|r|r|r|r|r|r|}
\hline & $\begin{array}{c}\mathrm{RT} \\
(\mathrm{min})\end{array}$ & $\begin{array}{c}\text { Area } \\
\left(\mathrm{V}^{*} \text { sec }\right)\end{array}$ & $\%$ Area & $\begin{array}{c}\text { Height } \\
(\mathrm{V})\end{array}$ & $\begin{array}{c}\% \\
\text { Height }\end{array}$ \\
\hline 1 & 3.364 & 26252 & 0.46 & 3450 & 1.30 \\
\hline 2 & 4.307 & 22782 & 0.40 & 1905 & 0.72 \\
\hline 3 & 4.639 & 63174 & 1.11 & 5158 & 1.94 \\
\hline 4 & 9.232 & 2709062 & 47.80 & 138219 & 51.93 \\
\hline 5 & 10.129 & 2812165 & 49.62 & 116031 & 43.59 \\
\hline 6 & 12.040 & 34209 & 0.60 & 1417 & 0.53 \\
\hline
\end{tabular}


<smiles>CC(=O)CC(O)C(C)C</smiles>

( $4 \mathbf{k}) 98 \%$ ee

\section{ulas}

Project Name: Defaults

Reported by User: System

\begin{tabular}{|llll|}
\hline & \multicolumn{2}{c|}{ S A M P L E } & I N F O R M A T I O N \\
\hline \hline & & & \\
Sample Name: & TZ-91 & Acquired By: & System \\
Sample Type: & Unknown & Date Acquired: & $1 / 20 / 031: 26: 02 \mathrm{PM}$ \\
Vial: & 1 & Acq. Method: & 3 uv2 280 \\
Injection \#: & 3 & Date Processed: $1 / 20 / 031: 38: 36 \mathrm{PM}$ \\
Injection Volume: & 20.00 ul & Channel Name: 2487 Channel 1 \\
Run Time: & 60.00 Minutes & Sample Set Name: \\
\hline
\end{tabular}

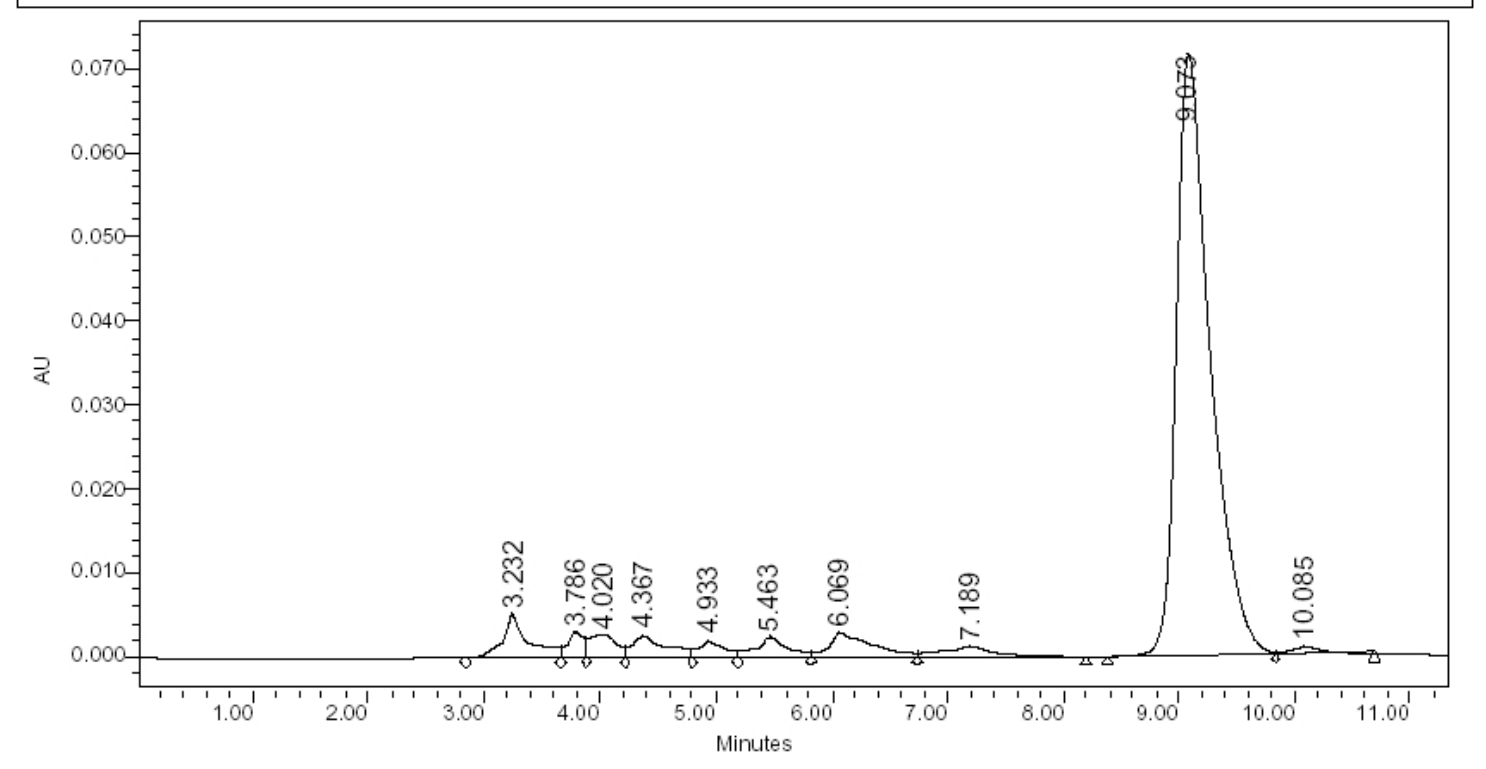

\begin{tabular}{|r|r|r|r|r|r|}
\hline & $\begin{array}{c}\text { RT } \\
(\mathrm{min})\end{array}$ & $\begin{array}{c}\text { Area } \\
\left(\mathrm{V}^{*} \mathrm{sec}\right)\end{array}$ & $\%$ Area & $\begin{array}{c}\text { Height } \\
(\mathrm{V})\end{array}$ & $\begin{array}{c}\% \\
\text { Height }\end{array}$ \\
\hline 1 & 3.232 & 82551 & 4.39 & 5153 & 5.44 \\
\hline 2 & 3.786 & 30071 & 1.60 & 3084 & 3.26 \\
\hline 3 & 4.020 & 43439 & 2.31 & 2734 & 2.89 \\
\hline 4 & 4.367 & 54886 & 2.92 & 2545 & 2.69 \\
\hline 5 & 4.933 & 28968 & 1.54 & 1866 & 1.97 \\
\hline 6 & 5.463 & 46504 & 2.47 & 2425 & 2.56 \\
\hline 7 & 6.069 & 75586 & 4.02 & 2956 & 3.12 \\
\hline 8 & 7.189 & 42076 & 2.24 & 1296 & 1.37 \\
\hline 9 & 9.073 & 1458137 & 77.54 & 71790 & 75.79 \\
\hline 10 & 10.085 & 18251 & 0.97 & 873 & 0.92 \\
\hline
\end{tabular}




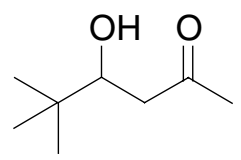

( 4I )-racemic

ulas

Project Name: Defaults

Reported by User: System

'Breezt

\begin{tabular}{|llll|}
\hline & \multicolumn{2}{c|}{ S A M P L E } & I N F O R M A T I O N \\
\hline \hline & & & \\
Sample Name: & TZ-160. & Acquired By: & System \\
Sample Type: & Unknown & Date Acquired: & $1 / 29 / 034: 16: 10 \mathrm{PM}$ \\
Vial: & 1 & Acq. Method: & 3 uv280 \\
Injection \#: & 6 & Date Processed: $1 / 30 / 039: 57: 41 \mathrm{AM}$ \\
Injection Volume: & $20.00 \mathrm{ul}$ & Channel Name: & 2487Channel 1 \\
Run Time: & 60.00 Minutes & Sample Set Name: \\
\hline
\end{tabular}

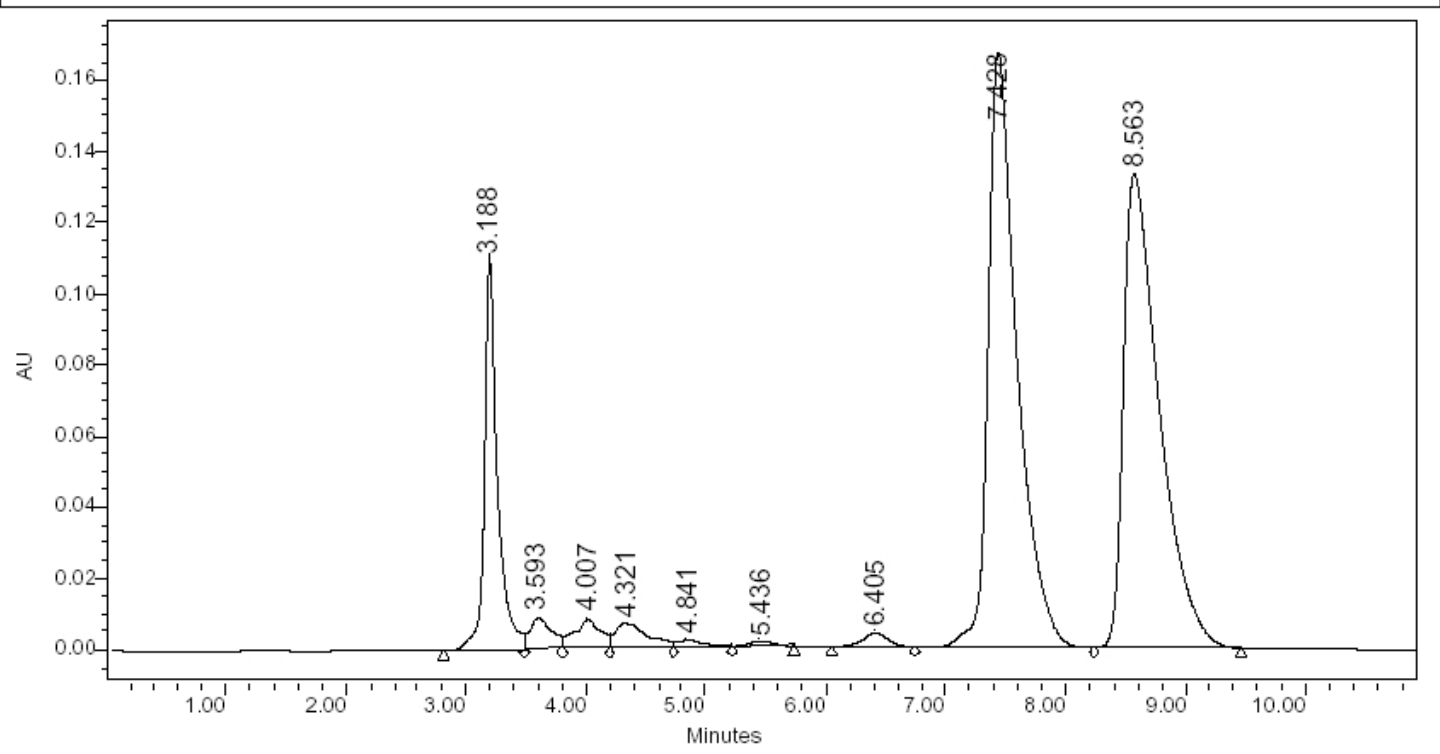

\begin{tabular}{|r|r|r|r|r|r|}
\hline & $\begin{array}{c}\text { RT } \\
(\mathrm{min})\end{array}$ & $\begin{array}{c}\text { Area } \\
\left(\mathrm{V}^{*} \mathrm{sec}\right)\end{array}$ & $\%$ Area & $\begin{array}{c}\text { Height } \\
(\mathrm{V})\end{array}$ & $\begin{array}{c}\% \\
\text { Height }\end{array}$ \\
\hline 1 & 3.188 & 830539 & 12.01 & 110749 & 24.93 \\
\hline 2 & 3.593 & 115884 & 1.68 & 9139 & 2.06 \\
\hline 3 & 4.007 & 131178 & 1.90 & 8481 & 1.91 \\
\hline 4 & 4.321 & 135598 & 1.96 & 7132 & 1.61 \\
\hline 5 & 4.841 & 37118 & 0.54 & 2358 & 0.53 \\
\hline 6 & 5.436 & 27667 & 0.40 & 1777 & 0.40 \\
\hline 7 & 6.405 & 64470 & 0.93 & 4116 & 0.93 \\
\hline 8 & 7.428 & 2813219 & 40.69 & 167371 & 37.67 \\
\hline 9 & 8.563 & 2758218 & 39.89 & 133185 & 29.98 \\
\hline
\end{tabular}




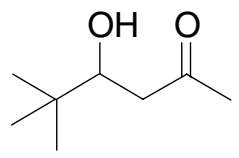

(4I ) $99 \%$ ee

ulas

Project Name: Defaults

'Breelt

Reported by User: System

\begin{tabular}{|llll|}
\hline & \multicolumn{2}{c|}{ S A M P L E } & I N F O R M A T I O N \\
\hline \hline & & & \\
Sample Name: & TZ-161. & Acquired By: & System \\
Sample Type: & Unknown & Date Acquired: & $1 / 30 / 039: 26: 28 \mathrm{AM}$ \\
Vial: & 1 & Acq. Method: & 3 uvv280 \\
Injection \#: & 1 & Date Processed: $1 / 30 / 039: 40: 17 \mathrm{AM}$ \\
Injection Volume: & $20.00 \mathrm{ul}$ & Channel Name: & 2487Channel 1 \\
Run Time: & 60.00 Minutes & Sample Set Name: \\
\end{tabular}

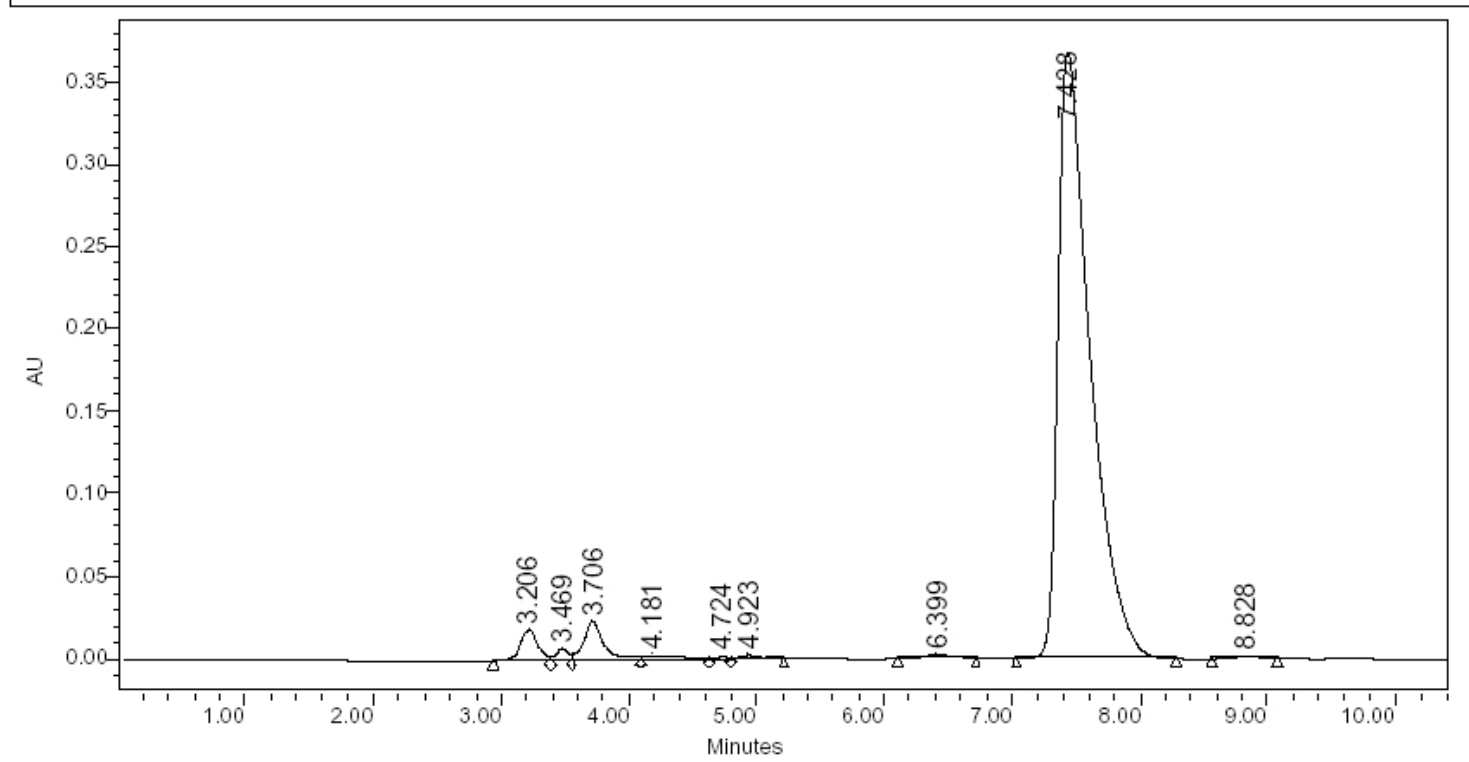

\begin{tabular}{|r|r|r|r|r|r|}
\hline & $\begin{array}{c}\text { RT } \\
(\mathrm{min})\end{array}$ & $\begin{array}{c}\text { Area } \\
\left(\mathrm{V}^{*} \mathrm{sec}\right)\end{array}$ & $\%$ Area & $\begin{array}{c}\text { Height } \\
(\mathrm{V})\end{array}$ & $\begin{array}{c}\% \\
\text { Height }\end{array}$ \\
\hline 1 & 3.206 & 176491 & 2.51 & 18279 & 4.28 \\
\hline 2 & 3.469 & 47706 & 0.68 & 7058 & 1.65 \\
\hline 3 & 3.706 & 255443 & 3.63 & 23529 & 5.51 \\
\hline 4 & 4.181 & 50173 & 0.71 & 2038 & 0.48 \\
\hline 5 & 4.724 & 10941 & 0.16 & 1225 & 0.29 \\
\hline 6 & 4.923 & 24839 & 0.35 & 2165 & 0.51 \\
\hline 7 & 6.399 & 33436 & 0.48 & 2654 & 0.62 \\
\hline 8 & 7.428 & 6413902 & 91.21 & 368376 & 86.33 \\
\hline 9 & 8.828 & 19244 & 0.27 & 1360 & 0.32 \\
\hline
\end{tabular}




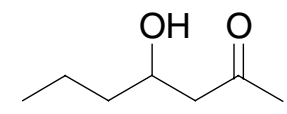

(4m)-racemic

\section{N2000 xT站}

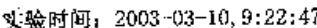

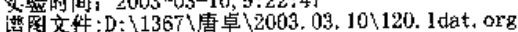

报告则间: 2003-03-12, 13:49:40

计算方法: 但积明法

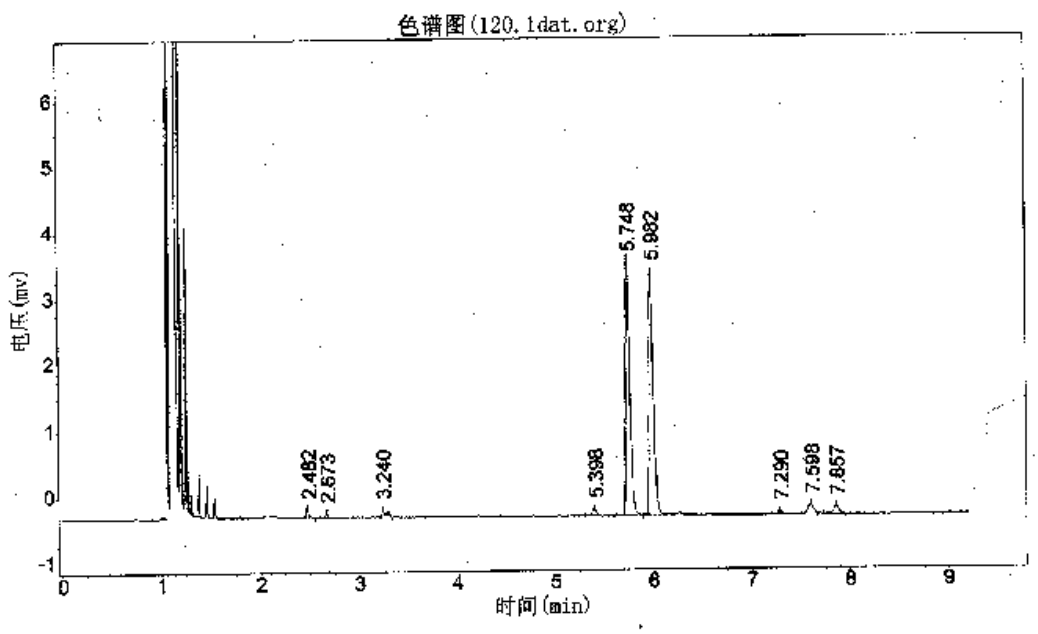

分析结果表

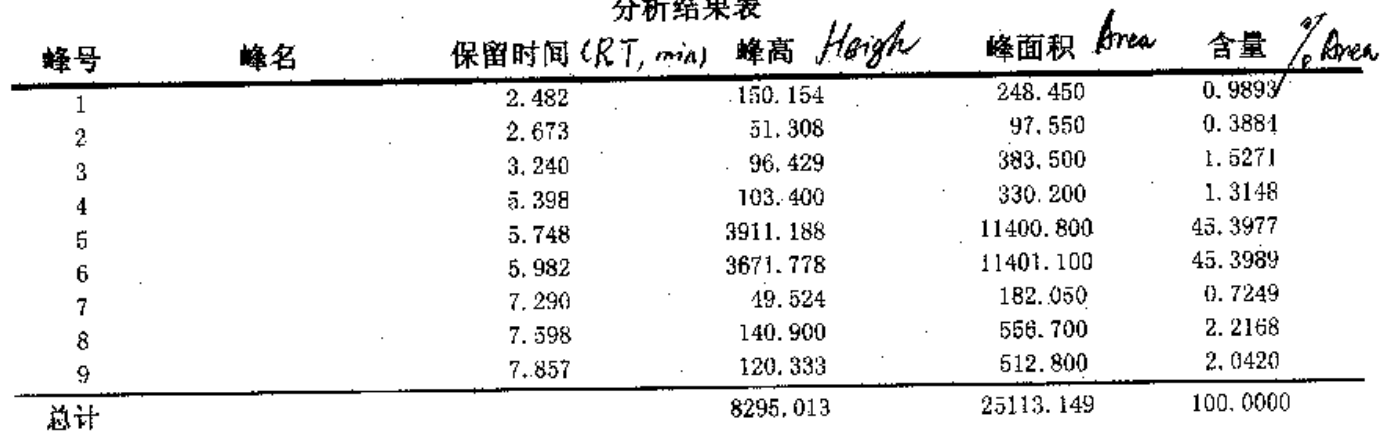




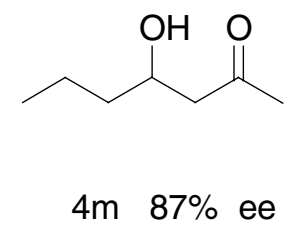

N2000 婮工作站

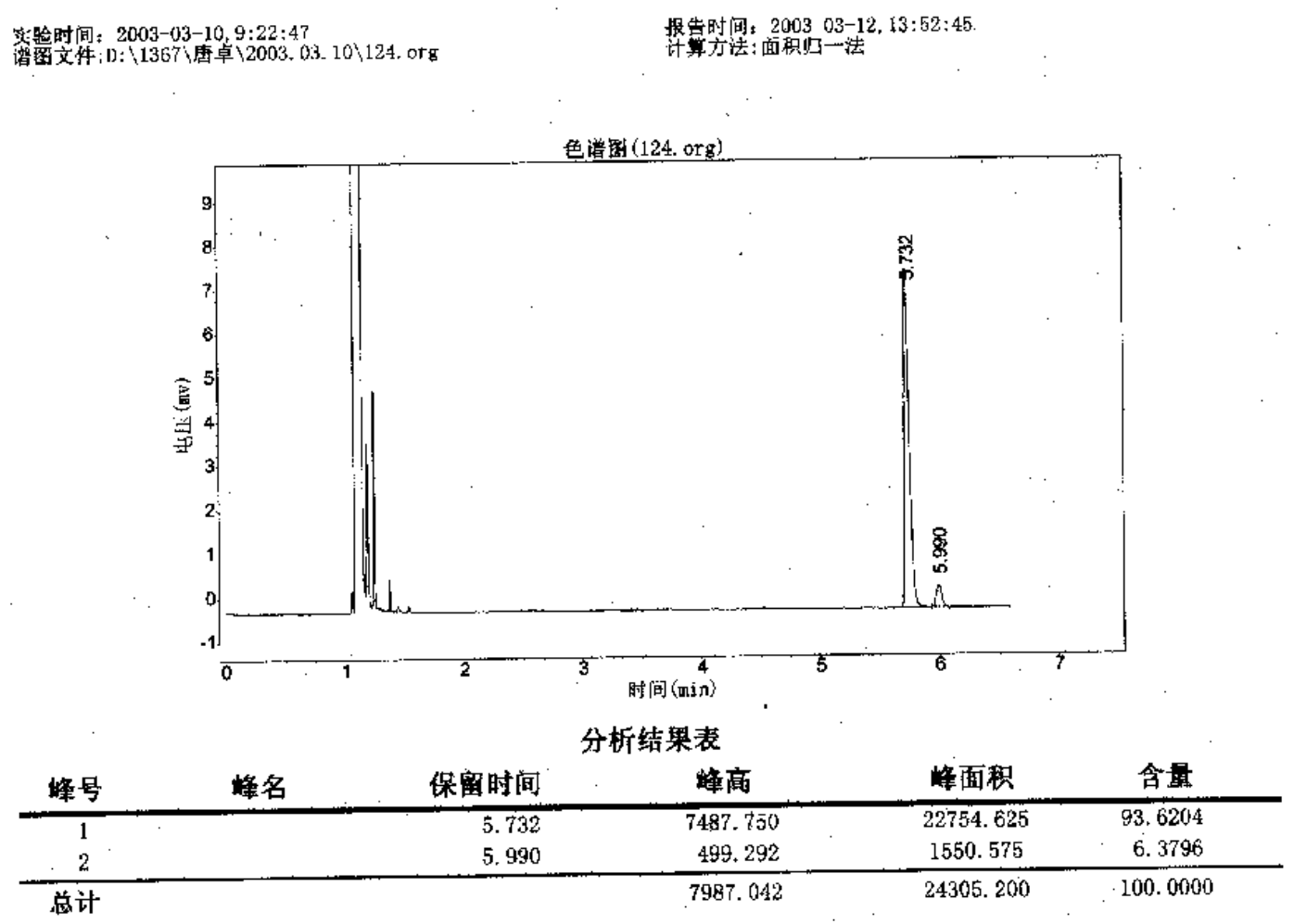




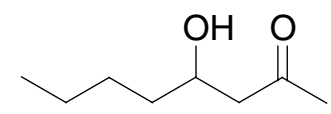

(4n)-racemic

\section{N2000 织工作站}

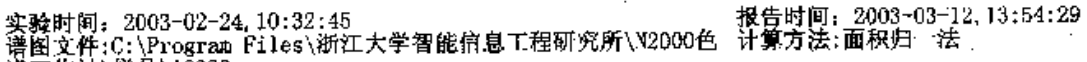
语工作站样器 10020 . org

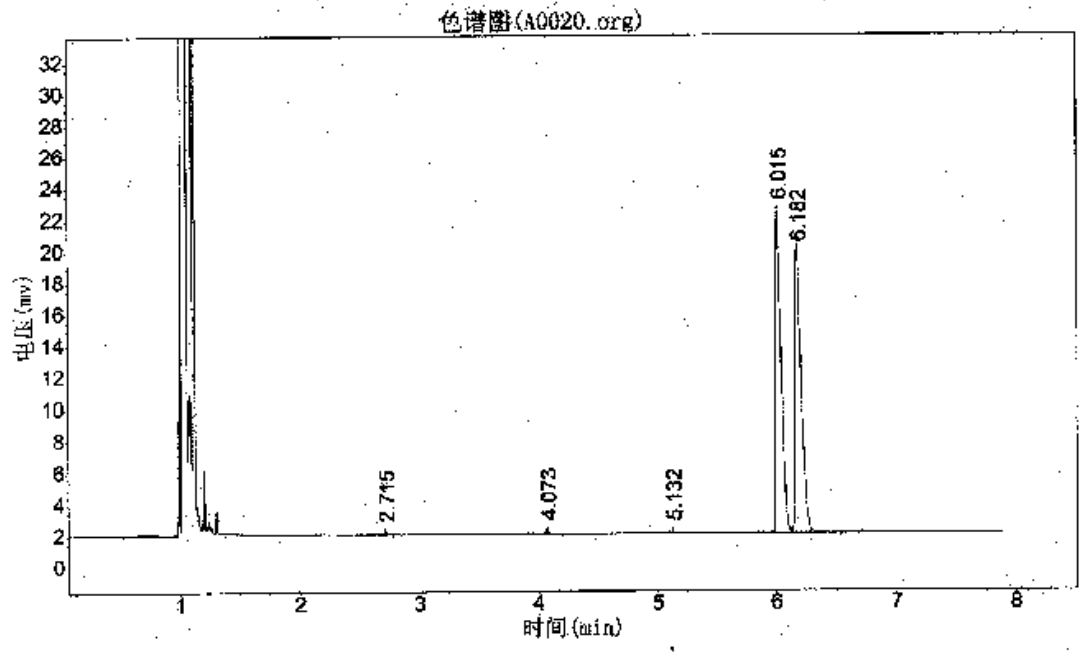

分析结果䒾

\begin{tabular}{|c|c|c|c|c|c|}
\hline 盽号 & 峰名 & 保罢䀦间 & 蜼符 & 峰面积 & 含量 \\
\hline 1 & & 2.715 & 51.216 & 324.550 & $0.2 \overline{5} 00$ \\
\hline 2 & & 4. 073 & 106.448 & 319.850 & 0.2464 \\
\hline 3 & & 5. 132 & 41.143 & 109.100 & 0.0843 \\
\hline 4 & & $6.01 \bar{\jmath}$ & 20172.383 & $6435 \% .105$ & 49.5814 \\
\hline 5 & & 6.182 & 17930.324 & fิ4687. 895 & 49.8378 \\
\hline 总计 & & & 38301.514 & $1297 \div 6.800$ & 100.0000 \\
\hline
\end{tabular}




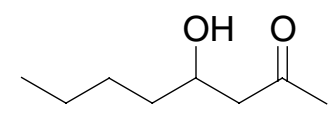

(4n) $86 \%$ ee

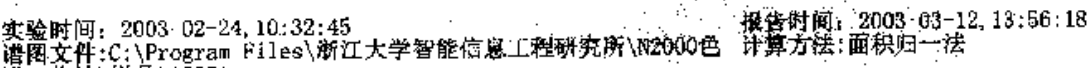
港下. 柞站 样品 10021. org

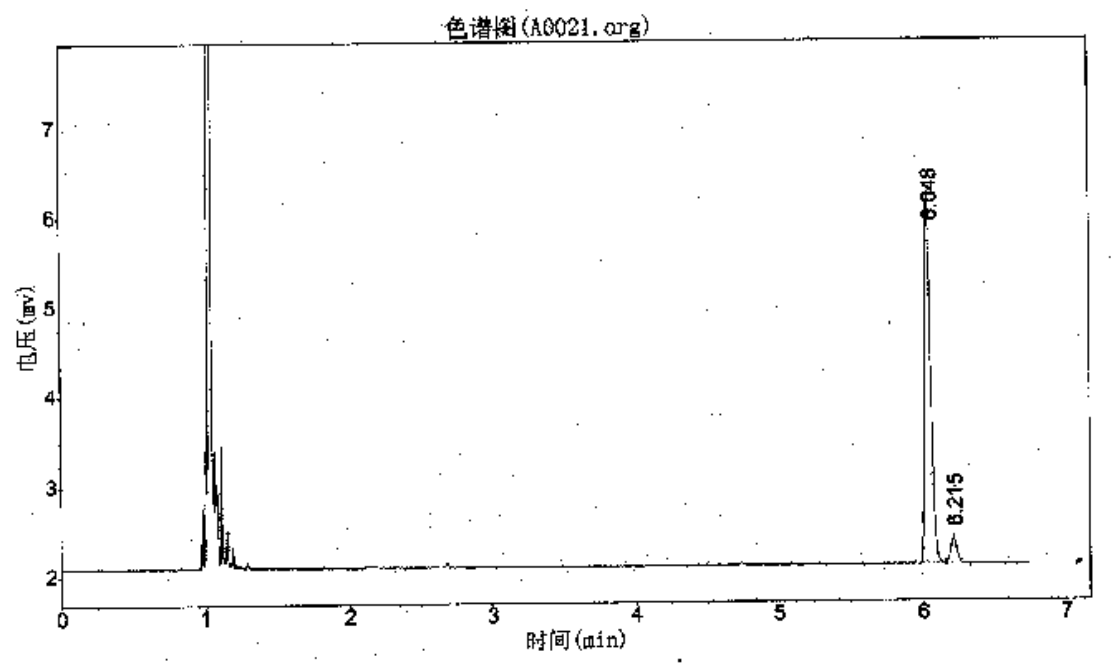

·分析结果表

\begin{tabular}{|c|c|c|c|c|c|}
\hline 峰客 & 蜂名 & 保兽肘荡 & 峰高 & 管面积 & 含量。 \\
\hline 1 & & 6.048 & 3967.522 & 12125.348 & 93.2024 \\
\hline 2 & & 6.215 & 262.391 & 884.362 & 6. 7976 \\
\hline 总计 & & & 4229.913 & 13009.700 & 100.0000 \\
\hline
\end{tabular}




\section{Calculations:}

Transition state geometries for the aldol reaction of benzaldehyde with acetone catalyzed by $\mathbf{3 d}$ :

Calculations were performed using the Gaussian 98 Program. ${ }^{3}$ Geometries were optimized by the Hartree-Fock method with the 6-31G* basis set and energies were further evaluated with the B3LYP/ 6-31G** method. ${ }^{4}$

The resulting Z-matrix for the two transition structures TS1 and TS2, as well as the total energies are the follows:

\section{Structure of TS1:}

$\mathrm{C}$

$\mathrm{N}$

$\mathrm{C}$

$\mathrm{C}$

$\mathrm{C}$

$\mathrm{C}$

$\mathrm{O}$

$\mathrm{N}$

$\mathrm{C}$

$\mathrm{C}$

$\mathrm{O}$

C

C

C

$\mathrm{O}$

C

C

C

$\mathrm{C}$

C

C

C

C
C

N

C

C

C

C

O

$\mathrm{N}$

C

O

C

C

C

$\mathrm{O}$

C

$\mathrm{C}$

C

C

19

20

21

C

$\begin{array}{r}1 \\ 2 \\ 3 \\ 4 \\ 3 \\ 6 \\ 6 \\ 8 \\ 9 \\ 10 \\ 2 \\ 12 \\ 13 \\ 14 \\ 12 \\ 9 \\ 17 \\ 18 \\ 19 \\ 20 \\ 21 \\ 10 \\ \hline\end{array}$

\subsection{9}

$1.470643 \quad 1 \quad 111.535694$

$\begin{array}{lllll}1.534105 & 2 & 102.726629 & 1 & -18.959757\end{array}$

$\begin{array}{lllll}1.528336 & 3 & 103.070632 & 2 & 34.918845\end{array}$

$\begin{array}{lllll}1.534329 & 2 & 116.131033 & 1 & 101.713701\end{array}$

$\begin{array}{lllll}1.204588 & 3 & 116.767732 & 2 & 169.650086\end{array}$

$\begin{array}{lllll}1.334317 & 3 & 118.128003 & 2 & -12.183091\end{array}$

$\begin{array}{lllll}1.459204 & 6 & 120.560648 & 3 & 178.377591\end{array}$

$\begin{array}{lllll}1.552064 & 8 & 109.293758 & 6 & -152.835696\end{array}$

$\begin{array}{lllll}1.397230 & 9 & 110.128042 & 8 & 46.675975\end{array}$

$\begin{array}{lllll}1.310448 & 1 & 122.885195 & 5 & 179.528346\end{array}$

$\begin{array}{lllll}1.413034 & 2 & 121.896067 & 1 & -8.155880\end{array}$

$\begin{array}{lllll}1.936933 & 12 & 100.746965 & 2 & -88.235265\end{array}$

$\begin{array}{lllll}1.269959 & 13 & 110.680921 & 12 & 39.671722\end{array}$

$\begin{array}{lllll}1.504950 & 2 & 118.762154 & 1 & 179.797088\end{array}$

$\begin{array}{lllll}1.522052 & 8 & 111.253186 & 6 & 82.158132\end{array}$

$\begin{array}{lllll}1.386589 & 9 & 120.690498 & 8 & -125.648804\end{array}$

$\begin{array}{lllll}1.386879 & 17 & 120.818918 & 9 & -179.263721\end{array}$

$\begin{array}{lllll}1.383614 & 18 & 120.206318 & 17 & -0.128545\end{array}$

$\begin{array}{lllll}1.386949 & 19 & 119.499381 & 18 & -0.110859\end{array}$

$\begin{array}{lllll}1.383935 & 20 & 120.097914 & 19 & 0.101806\end{array}$

$\begin{array}{lllll}1.518329 & 9 & 111.152075 & 8 & 167.709171\end{array}$ 


\begin{tabular}{|c|c|c|c|c|c|c|}
\hline C & 23 & 1.391069 & 10 & 120.069430 & 9 & -84.587315 \\
\hline $\mathrm{C}$ & 24 & 1.383800 & 23 & 120.596045 & 10 & 179.367845 \\
\hline $\mathrm{C}$ & 25 & 1.386857 & 24 & 120.262101 & 23 & 0.150354 \\
\hline $\mathrm{C}$ & 26 & 1.383562 & 25 & 119.517316 & 24 & 0.146646 \\
\hline C & 27 & 1.386875 & 26 & 120.114801 & 25 & -0.131128 \\
\hline $\mathrm{C}$ & 14 & 1.511860 & 13 & 100.036197 & 12 & 165.379580 \\
\hline $\mathrm{C}$ & 29 & 1.389672 & 14 & 121.144536 & 13 & 74.649535 \\
\hline C & 30 & 1.384162 & 29 & 120.634360 & 14 & 178.924718 \\
\hline $\mathrm{C}$ & 31 & 1.386578 & 30 & 119.961427 & 29 & -0.179195 \\
\hline C & 32 & 1.385726 & 31 & 119.683985 & 30 & -0.422433 \\
\hline C & 33 & 1.385570 & 32 & 120.251798 & 31 & 0.330698 \\
\hline $\mathrm{H}$ & 8 & 1.002735 & 6 & 119.714262 & 3 & 9.729167 \\
\hline $\mathrm{H}$ & 11 & 0.957700 & 10 & 107.334635 & 9 & -86.736520 \\
\hline $\mathrm{H}$ & 14 & 1.091465 & 13 & 95.275608 & 12 & -82.334548 \\
\hline $\mathrm{H}$ & 13 & 1.076519 & 12 & 114.641509 & 2 & 159.839664 \\
\hline $\mathrm{H}$ & 9 & 1.080832 & 8 & 107.063882 & 6 & -36.362688 \\
\hline $\mathrm{H}$ & 10 & 1.085880 & 9 & 108.404347 & 8 & -73.062204 \\
\hline $\mathrm{H}$ & 3 & 1.078590 & 2 & 110.658186 & 1 & -137.712385 \\
\hline $\mathrm{H}$ & 4 & 1.085418 & 3 & 109.248789 & 2 & -82.483884 \\
\hline $\mathrm{H}$ & 4 & 1.080130 & 3 & 111.431416 & 2 & 157.765151 \\
\hline $\mathrm{H}$ & 1 & 1.077160 & 2 & 109.664948 & 12 & 57.898322 \\
\hline $\mathrm{H}$ & 1 & 1.084477 & 2 & 108.791540 & 12 & -61.481411 \\
\hline $\mathrm{H}$ & 5 & 1.083384 & 4 & 110.261273 & 3 & 79.779716 \\
\hline $\mathrm{H}$ & 5 & 1.082728 & 4 & 113.012600 & 3 & -159.298504 \\
\hline $\mathrm{H}$ & 13 & 1.073990 & 12 & 117.182349 & 2 & 23.742007 \\
\hline $\mathrm{H}$ & 16 & 1.079602 & 12 & 110.155128 & 2 & -179.797232 \\
\hline $\mathrm{H}$ & 16 & 1.084787 & 12 & 109.983925 & 2 & 60.624005 \\
\hline $\mathrm{H}$ & 16 & 1.083719 & 12 & 111.274842 & 2 & -59.499390 \\
\hline $\mathrm{H}$ & 18 & 1.074967 & 17 & 119.497555 & 9 & 1.084936 \\
\hline $\mathrm{H}$ & 19 & 1.075555 & 18 & 119.691513 & 17 & -179.877815 \\
\hline $\mathrm{H}$ & 20 & 1.075612 & 19 & 120.286781 & 18 & -179.919682 \\
\hline $\mathrm{H}$ & 21 & 1.075950 & 20 & 120.067386 & 19 & -179.898719 \\
\hline $\mathrm{H}$ & 22 & 1.076413 & 21 & 119.413160 & 20 & -179.666517 \\
\hline
\end{tabular}




$\begin{array}{lrrrrrr}\mathrm{H} & 24 & 1.073924 & 23 & 119.330167 & 10 & -1.756530 \\ \mathrm{H} & 25 & 1.075884 & 24 & 119.732953 & 23 & 179.801648 \\ \mathrm{H} & 26 & 1.075722 & 25 & 120.228403 & 24 & 179.823931 \\ \mathrm{H} & 27 & 1.075684 & 26 & 120.167696 & 25 & 179.643157 \\ \mathrm{H} & 28 & 1.075534 & 27 & 119.461889 & 26 & 179.991570 \\ \mathrm{H} & 30 & 1.077196 & 29 & 119.793749 & 14 & -0.620139 \\ \mathrm{H} & 31 & 1.075615 & 30 & 119.939627 & 29 & -179.784775 \\ \mathrm{H} & 32 & 1.075524 & 31 & 120.111656 & 30 & -179.822909 \\ \mathrm{H} & 33 & 1.075800 & 32 & 119.932532 & 31 & -179.077915 \\ \mathrm{H} & 34 & 1.073235 & 33 & 120.896045 & 32 & -178.336856\end{array}$

$\mathrm{E}\left(\mathrm{HF} / 6-31 \mathrm{G}^{*}\right)=\mathbf{- \mathbf { 1 4 5 0 }} \mathbf{2 6 0 8 4}$ a.u.

$\mathrm{E}\left(\mathrm{B} 3 \mathrm{LYP} / 6-31 \mathrm{G}^{* *} / / \mathrm{HF} / 6-31 \mathrm{G}^{*}\right)=\mathbf{- 1 4 5 9 . 5 5 1 7 6}$ a.u.

\section{$\underline{\text { Structure of TS2: }}$}

C

$\mathrm{N}$

$\mathrm{C}$

$\mathrm{C}$

$\mathrm{C}$

$\mathrm{C}$

$\mathrm{O}$

$\mathrm{N}$

$\mathrm{C}$

$\mathrm{C}$

$\mathrm{O}$

$\mathrm{C}$

$\mathrm{C}$

$\mathrm{C}$

$\mathrm{O}$

$\mathrm{C}$

C

$\mathrm{C}$
1.476516

$\begin{array}{lll}1.472322 & 1 & 111.229006\end{array}$

$\begin{array}{lllll}1.532771 & 2 & 102.424035 & 1 & -21.535754\end{array}$

$\begin{array}{lllll}1.528700 & 3 & 102.781201 & 2 & 36.741685\end{array}$

$\begin{array}{lllll}1.535878 & 2 & 116.331827 & 1 & 98.667393\end{array}$

$\begin{array}{lllll}1.205049 & 3 & 116.345653 & 2 & 174.671401\end{array}$

$\begin{array}{lllll}1.332748 & 3 & 118.229209 & 2 & -6.945854\end{array}$

$\begin{array}{lllll}1.458747 & 6 & 120.885051 & 3 & 176.540828\end{array}$

$\begin{array}{lllll}1.553449 & 8 & 109.451001 & 6 & -141.183524\end{array}$

$\begin{array}{lllll}1.397797 & 9 & 109.967213 & 8 & 45.297059\end{array}$

$\begin{array}{lllll}1.306397 & 1 & 122.934878 & 5 & -179.599669\end{array}$

$\begin{array}{lllll}1.422896 & 2 & 122.207448 & 1 & -5.570958\end{array}$

$\begin{array}{lllll}1.927338 & 12 & 101.666588 & 2 & -85.699210\end{array}$

$\begin{array}{lllll}1.274763 & 13 & 111.397428 & 12 & 5.646536\end{array}$

$\begin{array}{lllll}1.502403 & 2 & 118.867914 & 1 & -178.524011\end{array}$

$\begin{array}{rrrrr}1.521375 & 8 & 111.142538 & 6 & 93.576173\end{array}$

$\begin{array}{lllll}1.386608 & 9 & 120.719976 & 8 & -125.220567\end{array}$ 


\begin{tabular}{|c|c|c|c|c|c|c|}
\hline C & 18 & 1.387390 & 17 & 120.804821 & 9 & -179.716102 \\
\hline C & 19 & 1.383322 & 18 & 120.193996 & 17 & -0.082861 \\
\hline $\mathrm{C}$ & 20 & 1.387389 & 19 & 119.513599 & 18 & -0.073861 \\
\hline $\mathrm{C}$ & 21 & 1.383611 & 20 & 120.123316 & 19 & 0.053924 \\
\hline C & 10 & 1.517776 & 9 & 111.061845 & 8 & 166.270085 \\
\hline $\mathrm{C}$ & 23 & 1.391221 & 10 & 120.133690 & 9 & -83.948288 \\
\hline $\mathrm{C}$ & 24 & 1.383728 & 23 & 120.587839 & 10 & 179.304802 \\
\hline $\mathrm{C}$ & 25 & 1.387001 & 24 & 120.264023 & 23 & 0.102410 \\
\hline $\mathrm{C}$ & 26 & 1.383520 & 25 & 119.522661 & 24 & 0.151790 \\
\hline C & 27 & 1.386997 & 26 & 120.109237 & 25 & -0.101258 \\
\hline C & 14 & 1.514629 & 13 & 102.327271 & 12 & -122.659996 \\
\hline $\mathrm{C}$ & 29 & 1.386888 & 14 & 120.963869 & 13 & 113.173895 \\
\hline $\mathrm{C}$ & 30 & 1.388190 & 29 & 120.366631 & 14 & 178.742036 \\
\hline $\mathrm{C}$ & 31 & 1.383381 & 30 & 120.420301 & 29 & -0.480485 \\
\hline $\mathrm{C}$ & 32 & 1.388239 & 31 & 119.558503 & 30 & -0.327529 \\
\hline $\mathrm{C}$ & 33 & 1.381973 & 32 & 119.917793 & 31 & 0.434761 \\
\hline $\mathrm{H}$ & 8 & 1.003674 & 6 & 119.967011 & 3 & 8.890176 \\
\hline $\mathrm{H}$ & 11 & 0.957459 & 10 & 107.021781 & 9 & -84.918001 \\
\hline $\mathrm{H}$ & 13 & 1.075096 & 12 & 113.191309 & 2 & 159.259452 \\
\hline $\mathrm{H}$ & 9 & 1.079502 & 8 & 106.557457 & 6 & -24.769979 \\
\hline $\mathrm{H}$ & 10 & 1.084908 & 9 & 108.564898 & 8 & -74.264987 \\
\hline $\mathrm{H}$ & 3 & 1.078721 & 2 & 110.435791 & 1 & -140.465760 \\
\hline $\mathrm{H}$ & 4 & 1.085486 & 3 & 109.396034 & 2 & -80.648847 \\
\hline $\mathrm{H}$ & 4 & 1.079578 & 3 & 111.360062 & 2 & 159.354800 \\
\hline $\mathrm{H}$ & 1 & 1.079663 & 2 & 109.745772 & 12 & 58.823441 \\
\hline $\mathrm{H}$ & 1 & 1.083463 & 2 & 109.001326 & 12 & -60.355837 \\
\hline $\mathrm{H}$ & 5 & 1.083514 & 4 & 110.287401 & 3 & 79.269741 \\
\hline $\mathrm{H}$ & 5 & 1.082596 & 4 & 113.118896 & 3 & -159.700821 \\
\hline $\mathrm{H}$ & 13 & 1.076950 & 12 & 116.053712 & 2 & 27.377881 \\
\hline $\mathrm{H}$ & 16 & 1.079051 & 12 & 109.964039 & 2 & 174.261222 \\
\hline $\mathrm{H}$ & 16 & 1.083066 & 12 & 110.404117 & 2 & 54.578371 \\
\hline $\mathrm{H}$ & 16 & 1.085069 & 12 & 110.570420 & 2 & -65.760680 \\
\hline $\mathrm{H}$ & 14 & 1.090207 & 13 & 93.824566 & 12 & 125.935362 \\
\hline
\end{tabular}




$\begin{array}{lrrrrrr}\mathrm{H} & 18 & 1.075143 & 17 & 119.512782 & 9 & 0.501247 \\ \mathrm{H} & 19 & 1.075578 & 18 & 119.695536 & 17 & -179.911154 \\ \mathrm{H} & 20 & 1.075615 & 19 & 120.283475 & 18 & -179.961543 \\ \mathrm{H} & 21 & 1.075943 & 20 & 120.048032 & 19 & -179.989702 \\ \mathrm{H} & 22 & 1.076557 & 21 & 119.479713 & 20 & -179.755774 \\ \mathrm{H} & 24 & 1.074008 & 23 & 119.327735 & 10 & -1.751066 \\ \mathrm{H} & 25 & 1.075890 & 24 & 119.734018 & 23 & 179.799830 \\ \mathrm{H} & 26 & 1.075738 & 25 & 120.223949 & 24 & 179.849169 \\ \mathrm{H} & 27 & 1.075708 & 26 & 120.166311 & 25 & 179.653718 \\ \mathrm{H} & 28 & 1.075605 & 27 & 119.486364 & 26 & 179.931087 \\ \mathrm{H} & 30 & 1.071997 & 29 & 119.860559 & 14 & 0.116020 \\ \mathrm{H} & 31 & 1.075595 & 30 & 119.550803 & 29 & -179.874041 \\ \mathrm{H} & 32 & 1.075527 & 31 & 120.278179 & 30 & -179.728159 \\ \mathrm{H} & 33 & 1.075741 & 32 & 120.113098 & 31 & -179.199371 \\ \mathrm{H} & 34 & 1.077523 & 33 & 119.508781 & 32 & -179.660393\end{array}$

$\mathrm{E}\left(\mathrm{HF} / 6-31 \mathrm{G}^{*}\right)=-1450.25698$ a.u.

$\mathrm{E}\left(\mathrm{B} 3 \mathrm{LYP} / 6-31 \mathrm{G}^{* *} / / \mathrm{HF} / 6-31 \mathrm{G}^{*}\right)=-1459.54693$ a.u.

\section{Reference:}

(1) For synthesis of proline amide, see: (a) Rhyoo, H. Y.; Yoon, Y. A.; Park, H. J.; Chung, Y. K. Tetrahedron Lett. 2001, 42, 5045-5048. (b) Corma, A.; Iglesias, M.; Pino deI, C; Shánchez, F. J. Organomet. Chem. 1992, 431, 233-246. (c) Mucaiyama, T. Tetrahedron 1981, 37, 4111-4119. (d) Camona, A.; Corma, A.; Iglesias, M.; San José.A.; Shánchez, F. J. Organomet. Chem. 1995, 492, $11-21$.

(2) (a) List, B.; Lerner, R. A.; Barbas III, C. F. J. Am. Chem. Soc. 2000, 122, 2395. (b) Sakthivel, K.; Notz, W.; Bui, T.; Barbas III, C. F. J. Am. Chem. Soc. 2001, 123, 5260.

(3) Gaussian 98, (Revision A.7): Frisch, M. J.; Trucks, G. W.; Schlegel, H. B.; Scuseria, G. E.; Robb, M. A.; Cheeseman, J. R.; Zakrzewski, V. G.; Montgomery, J. A. Jr.; Stratmann, R. E.; Burant, J. C.; Dapprich, S.; Millam, J. M.; Daniels, A. D.; Kudin, K. N.; Strain, M. C.; Farkas, O.; Tomasi, J.; Barone, V.; Cossi, M.; Cammi, R.; Mennucci, B.; Pomelli, C.; Adamo, C.; Clifford, S.; Ochterski, J.; Petersson, G. A.; Ayala, P. Y.; Cui, Q.; Morokuma, K.; Malick, D. K.; Rabuck, A. D.; Raghavachari, 
K.; Foresman, J. B.; Cioslowski, J.; Ortiz, J. V.; Baboul, A. G.; Stefanov, B. B.; Liu, G.; Liashenko, A.; Piskorz, P.; Komaromi, I.; Gomperts, R.; Martin, R. L.; Fox, D. J.; Keith, T.; Al-Laham, M. A.; Peng, C. Y.; Nanayakkara, A.; Gonzalez, C.; Challacombe, M.; Gill, P. M. W.; Johnson, B.; Chen, W.; Wong, M. W.; Andres, J. L.; Gonzalez, C.; Head-Gordon, M.; Replogle, E. S.; Pople, J. A. Gaussian, Inc., Pittsburgh PA, 1998.

(4) Becke, A. D. J. Chem. Phys. 1993, 98, 5648. (b) Lee, C.; Yang, W.; Parr, R. G. Phys. Rev. B 37, 1988, 785. 